\title{
New period-luminosity and period-color relations of classical Cepheids
}

\section{Cepheids in LMC}

\author{
A. Sandage ${ }^{1}$, G. A. Tammann ${ }^{2}$, and B. Reindl ${ }^{2}$ \\ 1 The Observatories of the Carnegie Institution of Washington, 813 Santa Barbara Street, Pasadena, CA 91101, USA \\ 2 Astronomisches Institut der Universität Basel, Venusstrasse 7, 4102 Binningen, Switzerland \\ e-mail: G-A.Tammann@unibas.ch
}

Received 9 February 2004 / Accepted 11 May 2004

\begin{abstract}
Photometric data for 593 Cepheids in the LMC, measured by Udalski et al. in the OGLE survey, augmented by 97 longer period Cepheids from other sources, are analyzed for the period-color (P-C) and period-luminosity (P-L) relations, and for the variations of amplitude, light curve shape, and period across the instability strip at constant absolute magnitude. Both the P-C and P-L relations have different slopes for periods smaller and larger than 10 days. The break at 10 days is also seen in the period-amplitude relations, and the compound Fourier combinations of $R_{21}$ and $\Phi_{21}$ introduced by Simon and Lee. The LMC Cepheids are bluer than Galactic Cepheids in the $B, V$, and $I$ color bands, part of which is due to differential Fraunhofer line blanketing and part to real differences in the temperature boundaries of the instability strip. The LMC strip is hotter by between $80 \mathrm{~K}$ and $350 \mathrm{~K}$ depending on the period. Hence, both the slopes and (necessarily) the zero points of the $\mathrm{P}$-L relations in $B, V$, and $I$ must differ between LMC and the revised relations (also given here) for the Galaxy, and in fact they do. The LMC Cepheids are brighter by up to $0.5 \mathrm{mag}$ at $\log P=0.4$ ( 2 days) and fainter by $0.2 \mathrm{mag}$ at $\log P=1.5$ (32 days). These facts complicate the use of Cepheid as precision distance indicators until the reason is found (metallicity differences or other unknown differences) for the non-universality of the P-L and P-C relations.

The very large data base permits mapping of various Cepheid properties at different positions within the instability strip, both at constant period and at constant absolute magnitude over the range of $2<P<40$ days and $-2>M_{V}>-5$. Amplitude of the light curves are largest near the blue edge of the strip for periods between 2 and 7 days and longer than 15 days. The sense is reversed for periods between 7 and 15 days. The shape of the light curves varies systematically across the strip. Highly peaked curves (of large amplitude) that necessarily have large values of $R_{21}$ of about 0.5 , occur near the blue edge of the strip. More symmetrical (small amplitude) light curves that have, thereby, small values of $R_{21}$, generally occur near the red edge of the strip. Consequently, there is a strong correlation of $R_{21}$ with color within the strip at a given absolute magnitude. Strong correlations also exist between color and period, and color and amplitude at given absolute magnitudes, for the same reason that has long been known for RR Lyrae stars, based on the sloping lines of constant period in the CMD combined with the variation of amplitude, and now $R_{21}$, with color. The highly peaked light-curve shapes and large amplitudes (indicating a non-linear regime that is overdriven out of the linear regime) near the blue edge of the strip, show that the energy driver for the pulsation (i.e. the negative dissipation) is strongest at the blue edge.
\end{abstract}

Key words. stars: variables: Cepheids - galaxies: Magellanic Clouds - cosmology: distance scale

\section{Introduction}

We showed in Paper I (Tammann et al. 2003) that the periodluminosity (P-L) relation for Cepheids in the Galaxy, LMC, and SMC have significantly different slopes. If true, by necessity the slopes of the ridge-line (mean) period-color (P-C) relations in the three galaxies must also differ. We saw that this requirement was met, based on the extensive new photometric data by Udalski et al. $(1999 \mathrm{~b}, \mathrm{c})$ for LMC and SMC (Paper I, Fig. 16; and Sect. 4.2.2 here) from the OGLE project and that of Berdnikov et al. (2000) for the Galaxy.
The problem posed by this result is severe. With the calibrated Galactic P-L relation (as revised in Sect. 4.2.1 here) fixed by two independent methods (main sequence fittings and the Baade-Becker-Wesselink (BBW) kinematic expansion method), and in the LMC by its distance determined by a variety of non-Cepheid methods (Paper I, Table 6), our LMC relation is brighter than that for the Galaxy by -0.37 in $V$ at $\log P=0.4\left(P=2.5^{\mathrm{d}}\right)$, and fainter by $+0.0^{\mathrm{m}} 11$ at $\log P=$ $1.6\left(P=40^{\mathrm{d}}\right)$. The differences in $I$ at the same periods are -0.32 and $+0^{\mathrm{m}} .19$. The LMC and Galactic P-L relations 
cross at $\log P \approx 1.3\left(P=20^{\mathrm{d}}\right)$ if our adopted distance modulus of LMC at $(m-M)^{0}=18.54$ is correct.

A principal purpose for this series, of which this is the second paper of a projected four paper brief, is to analyze in tedious detail the new LMC and SMC data obtained by the OGLE consortium and to compare them with the Galaxy. Our purpose is to ferret out clues for the cause of the differences in the P-L and P-C relations and of the different position in the luminosity-temperature diagram of the Cepheids in the three galaxies. In the present discussion here we follow the methods of Paper I.

The prime suspects for at least part of the variations from galaxy to galaxy are the established metallicity differences, for the reasons suggested in Paper I. At the most elementary level, the purely technical effects on the colors of the differential blanketing effects for different metallicities affect the color-color relations, and color-period relations. And, as mentioned above, once there is a difference in the slopes of the color-period relations for whatever series of reasons, the $\mathrm{P}-\mathrm{L}$ relations must also differ.

We saw in Paper I that real temperature differences exist in the ridge-line $L_{\mathrm{bol}}, \log T_{\mathrm{e}}$ plane (the HR diagram) between the Galaxy and LMC Cepheids. Is this also due to the effect of metallicity differences in the much more complicated physics of the pulsation and the position of the instability strip? If so, this, of course, would be a deeper reason than the simple technical effect of Fraunhofer blanketing. One of the purposes of this paper is to extend the discussion of these points from Paper I here in Sect. 8.

A second purpose is to explore the properties of the instability strip by the same technique made manifest by the horizontal branch of globular clusters as it threads the instability strip nearly at constant luminosity. The large data base from the new precision photometric data of Udalski et al. (1999b,c) for the LMC Cepheids permit a variety of studies of the Cepheid properties as a function of position (color) in the strip, similar to such studies for the RR Lyrae stars.

Section 2 discusses the OGLE data of Udalski et al. (1999b,c) for LMC Cepheids, defining the sample, the Cepheid reddenings, and the errors. The period-color relations in $(B-$ $V)^{0}$ and $(V-I)^{0}$, with comparisons with the Galaxy, are in Sect. 3. The P-L relations for LMC in $B, V$, and $I$, with comparisons with an updated calibration of the Galactic Cepheids are in Sect. 4. Discussion of the break in the P-L and P-C relations at $\log P=1.0$, and the abnormal behavior of the Fourier components and amplitudes of the light-curves, also at 10 days, is in Sect. 5. Section 6 is an extended discussion of the position of the instability strip in the HR diagram $\left(M_{V}\right.$-color plane). Comparison with the Galaxy (Fig. 15 of Paper I) is made there. The break in the slope of the $M_{V}$-color relations in $(B-V)^{0}$ and $(V-I)^{0}$ at 10 days period is manifest. Also in that section, the slopes of the lines of constant period in the color-magnitude diagram (CMD) are derived in different period ranges, as are the correlations of amplitude with position (color) in the strip.

Section 7 shows the period-luminosity-color (PLC) relations in $B, V$, and $I$, based on these aforementioned properties of the LMC Cepheids. The instability strip in the luminositytemperature $\left(\log L-\log T_{\mathrm{e}}\right)$ plane is derived in Sect. 8 where the effects of different metallicities and helium abundances, based on models in the literature, are discussed. Section 9 is a summary, and states the dilemma caused by these results in the using of Cepheids as precision distance indicators in our program to calibrate the Hubble constant by means of Cepheid distances to galaxies that have produced type Ia supernovae (Parodi et al. 2000; Saha et al. 2001).

\section{The data for LMC Cepheids}

\subsection{The OGLE sample}

Udalski et al. (1999b) ${ }^{1}$ have presented multi-epoch CCD photometry in the standard $B V I$ system, obtained within the OGLE program, of hundreds of variable stars distributed along the bar of LMC and have identified those which they consider as classical, fundamental-mode Cepheids. After exclusion of double-entries we have also excluded, following a scrutiny kindly provided by S. Kanbur \& C.-C. Ngeow, 80 stars of the sample which have not equally well defined or atypical light curves. We have finally excluded 14 Cepheids with $\log P<1$ and being redder than $(B-V)^{0}>0.8$ or $(V-I)^{0}>0.9^{2}$. At least some of these stars may be type II Cepheids. This leaves 593 Cepheids with VI magnitudes, 584 of which have also $B$ magnitudes. Their period interval is restricted to $0.4 \leq$ $\log P \leq 1.5$. The lower limit is set, following Udalski et al. (1999b), to avoid confusion with stars which may not be classical, fundamental-mode Cepheids. The upper limit is forced by saturation effects of the OGLE observations. The clipped sample of Udalski et al. (1999b) is called U99 sample in the following.

\subsection{The additional sample}

Mainly because of the upper period limitation due to image saturation, the U99 sample was augmented by 97 LMC Cepheids for which photoelectric $V$ photometry could be compiled in the literature. From the numerous CCD magnitudes in $B, V$, and $I$ by Sebo et al. (2002) only those 20 Cepheids above $P=$ 10 days were retained which have at least ten or more single observations in each color. The very red and faint Cepheid HV 2749 is probably a type II Cepheid and is excluded. - The additional 97 Cepheids are listed in Table 1; 96 have $B, 97 \mathrm{~V}$, and $55 \mathrm{I}$ magnitudes. These Cepheids are referred to as the "Table 1 sample" in the following.

\subsection{The color excesses $E(B-V)$}

Individual color excesses for the U99 sample were adopted from Udalski et al. (1999b). These authors have determined absorption values $A_{I}$ from the mean $I$-magnitudes of adjacent red-clump stars which makes them exceptionally independent of the Cepheids themselves, particularly as to a possible spread

\footnotetext{
1 The revised and in $B$ extensively augmented catalog (updated on Apr. 24, 2003) is available via ftp://bulge. princeton.edu/ogle/ogle2/var_stars/lmc/cep/catalog/

2 All magnitudes quoted in this paper are magnitudes $\langle x\rangle$ of intensity means. Colors $(x-y)$ mean $\langle x\rangle-\langle y\rangle$.
} 
in metallicity. They then assume $E(B-V)=A_{I} / 1.96$ which we adopt, although our absorption law requires $E(B-V)=A_{I} / 1.95$ (see below). The small difference is unconsequential because the zero point of $E(B-V)$ is tied by Udalski et al. (1999b) to several external fields in LMC.

Most of the U99 Cepheids have $0.10<E(B-V) \leq 0.16$ with a median value of 0.135 , but there are also 122 Cepheids with $0.16<E(B-V) \leq 0.20$. These values are higher than the standard value of $E(B-V)=0.10$ for LMC, but it is known that the bar is relatively dust-rich (Hodge 1972). We will return to this question in Sect. 2.4.

The reddenings of the Table 1 sample were compiled from the literature where available; in the remaining 37 cases $E(B-$ $V)=0.10$ was assumed. The average value of $E(B-V)$ for this sample is 0.09 .

To convert the adopted $E(B-V)$ s into $E(V-I) \mathrm{s}$ and into the corresponding absorption values $A_{B V I}$ we assume that LMC Cepheids follow the same absorption law as Galactic Cepheids (see Sect. 4.2.1).

\subsection{Errors}

The merging of the U99 and Table 1 samples raises the question whether the independent photometries, although nominally in the standard system, are compatible indeed. To test this question we have derived P-C relations in $(B-V)^{0}$ and $(V-I)^{0}$ and $\mathrm{P}-\mathrm{L}_{B V I}$ relations below (Eqs. (2), (3), (5), (6), and (10)-(15)) from the joint sample and have determined random and systematic deviations of either one sample from the mean relations. The differences were combined to give the color and magnitude differences between the two samples as shown in Table 2 .

The photometric agreement between the two samples is very good; the difference of $\sim 0.01$ is in all cases statistically insignificant. The two samples can therefore be combined without introducing any noticeable systematic errors into the photometry.

The agreement of the photometry of the two samples is even more astounding as the comparison is performed after the colors and magnitudes have been corrected for the adopted reddenings. This provides a strong test for the adopted values of $E(B-V)$ and confirms that the reddenings of the U99 Cepheids in the bar of LMC must be larger on average than of the widely distributed Cepheids in Table 1.

\section{The period-color relations of LMC}

\subsection{The period-color $(P-C)$ relation in $(B-V)^{0}$}

The reddening-corrected colors $(B-V)^{0}$ of the accepted 679 Cepheids with known $(B-V)^{0}$ colors from U99 and Table 1 are plotted against $\log P$ in Fig. 1 a.

A linear fit over all periods of the data in Fig. 1a gives $(N=$ 679)

$(B-V)^{0}=(0.358 \pm 0.011) \log P+(0.294 \pm 0.009), \sigma=0.09$ (1)

in good statistical agreement with earlier LMC P-C relations (Caldwell \& Coulson 1986; Laney \& Stobie 1994). However, the linear fit is not particularly good. A better fit is achieved if the Cepheids with $\log P \gtrless 1.0$ are separately fitted. One obtains then for $\log P<1(N=570)$ :

$(B-V)^{0}=(0.273 \pm 0.024) \log P+(0.345 \pm 0.015), \sigma=0.09(2)$

and for $\log P>1(N=109)$ :

$(B-V)^{0}=(0.426 \pm 0.039) \log P+(0.214 \pm 0.052), \sigma=0.10$. (3)

The slope of the long-period Cepheids is clearly steeper $(3.4 \sigma)$ than for those with $\log P<1.0$. The breaking point at $\log P=1$ is not well determined; it will be justified below. No attempt was made here - nor in the following cases - to smooth out the jump in color of 0.022 at $\log P=1$ between Eqs. (2) and (3); although it is within the statistical error, it could also hint at a physical effect.

The scatter of $\sigma=0$. 09 in Eq. (2) is mainly due to the intrinsic width of the instability strip. The scatter in Eq. (3) is only marginally larger.

\subsection{The $P-C$ relation in $(V-I)^{O}$}

647 accepted and dereddened LMC Cepheids with known ( $V-$ $I)^{0}$ colors from U99 and Table 1 define the P-C relation in $(V-$ $I)^{0}$ (Fig. 1b).

A linear fit over all periods yields $(N=647)$

$(V-I)^{0}=(0.256 \pm 0.010) \log P+(0.444 \pm 0.008), \sigma=0.08$. (4)

Yet the data in Fig. 1b are not well fit through the linear Eq. (4). It predicts Cepheids with $\log P<0.5$ to be redder by 0.05 and those at $\log P \sim 1.6$ to be bluer by 0.05 as they are actually observed on average. Indeed eye inspection already suggests that the $\mathrm{P}-\mathrm{C}$ relation has a rather abrupt change of slope near $\log P \approx 1.0$. If, as for the $(B-V)^{0}$ colors above, one fits two separate linear regressions for short- and long-period Cepheids, one obtains for $\log P<1(N=556)$ :

$(V-I)^{0}=(0.160 \pm 0.022) \log P+(0.501 \pm 0.014), \sigma=0.08$

and for $\log P>1(N=91)$ :

$(V-I)^{0}=(0.315 \pm 0.032) \log P+(0.380 \pm 0.042), \sigma=0.08$.

The short-period Cepheids define a much flatter P-C relation than long-period ones. The slope difference has a significance of $4.1 \sigma$. Another way of expressing this result is provided by an t-test which excludes the hypothesis that the single-line solution of Eq. (4) is an equally good fit as the two separate fits of Eqs. (5) and (6) with a probability of more than $99 \%$ (Kanbur \& Ngeow 2004). The steep slope of the P-C relation in $(V-I)^{0}$ of long-period LMC Cepheids has been anticipated by Caldwell $\&$ Coulson (1986).

\subsection{Comparison of the $L M C$ and Galactic $P-C$ relations}

The mean LMC P-C relations in $(B-V)^{0}$ (Eqs. (2) and (3)) and $(V-I)^{0}$ (Eqs. (5) and (6)) are repeated in the inserts of Figs. 1a,b and compared with the position of 321 Galactic 
Table 1. LMC Cepheids with photoelectric photometry.

\begin{tabular}{|c|c|c|c|c|c|c|c|c|c|c|c|c|}
\hline $\begin{array}{c}\text { Cepheid } \\
\text { (1) }\end{array}$ & $\begin{array}{c}\log P \\
(2)\end{array}$ & $\begin{array}{c}V \\
(3)\end{array}$ & $\begin{array}{c}(B-V) \\
(4)\end{array}$ & $\begin{array}{c}(V-I) \\
(5)\end{array}$ & $\begin{array}{c}E(B-V) \\
\quad(6)\end{array}$ & $\begin{array}{l}\text { Source } \\
(7)\end{array}$ & $\begin{array}{l}R_{V} \\
(8)\end{array}$ & $\begin{array}{l}V^{0} \\
(9)\end{array}$ & $\begin{array}{c}(B-V)^{0} \\
(10)\end{array}$ & $\begin{array}{c}(V-I)^{0} \\
(11)\end{array}$ & $\begin{array}{l}\mathcal{A}_{V} \\
(12)\end{array}$ & $\begin{array}{c}\mathcal{A}_{B} \\
\text { (13) }\end{array}$ \\
\hline ROB 44 & 0.408 & 16.36 & 0.58 & $\ldots$ & 0.10 & 1 & 3.13 & 16.05 & 0.48 & $\ldots$ & 0.80 & 1.45 \\
\hline HV 5541 & 0.415 & 16.06 & 0.56 & $\ldots$ & 0.06 & 3 & 3.12 & 15.87 & 0.50 & $\ldots$ & 0.91 & $\ldots$ \\
\hline ROB 24 & 0.429 & 16.22 & 0.51 & $\ldots$ & 0.10 & 1 & 3.10 & 15.91 & 0.41 & $\ldots$ & 1.20 & 1.57 \\
\hline HV 12225 & 0.478 & 16.22 & 0.66 & $\ldots$ & 0.06 & 1,3 & 3.17 & 16.03 & 0.60 & $\ldots$ & 0.50 & 0.63 \\
\hline HV 2353 & 0.492 & 15.37 & 0.53 & $\ldots$ & 0.10 & 1 & 3.11 & 15.06 & 0.43 & $\ldots$ & $\ldots$ & 0.69 \\
\hline ROB 25 & 0.529 & 16.03 & 0.60 & $\ldots$ & 0.10 & 1 & 3.14 & 15.72 & 0.50 & $\ldots$ & 0.52 & 0.70 \\
\hline HV 12765 & 0.535 & 15.29 & 0.57 & $\ldots$ & 0.10 & 1 & 3.13 & 14.98 & 0.47 & $\ldots$ & $\ldots$ & 0.57 \\
\hline HV 12747 & 0.556 & 15.76 & 0.56 & $\ldots$ & 0.06 & 1,3 & 3.12 & 15.57 & 0.50 & $\ldots$ & 0.37 & 0.70 \\
\hline ROB 29 & 0.569 & 15.80 & 0.58 & $\ldots$ & 0.10 & 1 & 3.13 & 15.49 & 0.48 & $\ldots$ & 0.54 & 0.57 \\
\hline HV 12720 & 0.635 & 15.74 & 0.63 & $\ldots$ & 0.10 & 1 & 3.16 & 15.42 & 0.53 & $\ldots$ & $\ldots$ & 1.50 \\
\hline HV 12869 & 0.653 & 15.06 & 0.62 & $\ldots$ & 0.10 & 1 & 3.15 & 14.74 & 0.52 & $\ldots$ & $\ldots$ & 0.80 \\
\hline ROB 22 & 0.669 & 15.55 & 0.70 & $\cdots$ & 0.10 & 1 & 3.19 & 15.23 & 0.60 & $\ldots$ & 0.82 & 1.42 \\
\hline HV 12231 & 0.677 & 15.43 & 0.64 & $\ldots$ & 0.10 & 1 & 3.16 & 15.11 & 0.54 & $\ldots$ & $\ldots$ & 1.27 \\
\hline HV 6093 & 0.680 & 15.35 & 0.64 & $\ldots$ & 0.06 & 1,3 & 3.16 & 15.16 & 0.58 & $\ldots$ & $\ldots$ & 1.16 \\
\hline HV 12077 & 0.703 & 15.30 & 0.61 & $\ldots$ & 0.10 & 1 & 3.15 & 14.99 & 0.51 & $\ldots$ & $\ldots$ & 1.00 \\
\hline HV 12079 & 0.841 & 14.94 & 0.66 & $\ldots$ & 0.10 & 1 & 3.17 & 14.62 & 0.56 & $\ldots$ & $\ldots$ & 1.14 \\
\hline HV 935 & 0.849 & 15.02 & 0.71 & 0.80 & 0.10 & 5 & 3.19 & 14.70 & 0.61 & 0.67 & 0.68 & $\ldots$ \\
\hline HV 1000 & 0.859 & 15.04 & 0.76 & 0.76 & 0.10 & 5 & 3.21 & 14.72 & 0.66 & 0.63 & 0.78 & $\ldots$ \\
\hline HV 12976 & 0.895 & 14.99 & 0.74 & $\ldots$ & 0.10 & 1 & 3.20 & 14.67 & 0.64 & $\ldots$ & $\ldots$ & 1.76 \\
\hline HV 5730 & 0.897 & 15.12 & 0.85 & 0.85 & 0.10 & 5 & 3.25 & 14.79 & 0.75 & 0.72 & 0.65 & $\ldots$ \\
\hline HV 6104 & 0.902 & 14.85 & 0.70 & 0.81 & 0.10 & 5 & 3.19 & 14.53 & 0.60 & 0.68 & 0.80 & $\ldots$ \\
\hline HV 12581 & 0.904 & 15.15 & 0.83 & 0.85 & 0.10 & 5 & 3.24 & 14.83 & 0.73 & 0.72 & 0.60 & $\ldots$ \\
\hline HV 12700 & 0.911 & 14.84 & 0.74 & 0.75 & 0.03 & $1,3,4$ & 3.20 & 14.74 & 0.71 & 0.71 & $\ldots$ & 0.90 \\
\hline HV 12823 & 0.919 & 14.57 & 0.55 & $\ldots$ & 0.10 & 1 & 3.12 & 14.26 & 0.45 & $\ldots$ & $\ldots$ & 1.13 \\
\hline HV 2738 & 0.920 & 14.66 & 0.86 & 0.61 & 0.10 & 5 & 3.26 & 14.33 & 0.76 & 0.48 & 0.85 & $\ldots$ \\
\hline HV 2854 & 0.936 & 14.64 & 0.72 & 0.78 & 0.05 & $1,3,4$ & 3.19 & 14.48 & 0.67 & 0.72 & $\ldots$ & 0.85 \\
\hline HV 2733 & 0.941 & 14.69 & 0.62 & 0.76 & 0.12 & $1,3,4$ & 3.15 & 14.31 & 0.50 & 0.61 & $\ldots$ & 0.66 \\
\hline HV 12452 & 0.941 & 14.79 & 0.71 & $\ldots$ & 0.10 & 1 & 3.19 & 14.47 & 0.61 & $\ldots$ & $\ldots$ & 1.33 \\
\hline HV 12717 & 0.947 & 14.69 & 0.70 & $\ldots$ & 0.10 & 1 & 3.19 & 14.37 & 0.60 & $\ldots$ & $\ldots$ & 1.25 \\
\hline HV 12816 & 0.960 & 14.53 & 0.56 & 0.72 & 0.08 & $1,3,4,6$ & 3.12 & 14.28 & 0.48 & 0.62 & 0.69 & 1.08 \\
\hline HV 971 & 0.968 & 14.43 & 0.68 & $\ldots$ & 0.10 & 1 & 3.18 & 14.11 & 0.58 & $\ldots$ & $\ldots$ & 1.02 \\
\hline HV 2510 & 0.973 & 14.85 & 0.71 & 0.77 & 0.10 & 5 & 3.19 & 14.53 & 0.61 & 0.64 & 0.85 & $\ldots$ \\
\hline HV 2301 & 0.978 & 13.94 & 0.84 & $\ldots$ & 0.10 & 1 & 3.25 & 13.62 & 0.74 & $\ldots$ & $\ldots$ & 0.80 \\
\hline HV 12474 & 0.993 & 14.65 & 0.69 & $\ldots$ & 0.10 & 1 & 3.18 & 14.33 & 0.59 & $\ldots$ & $\ldots$ & 0.87 \\
\hline HV 6105 & 1.019 & 14.91 & 0.79 & $\ldots$ & 0.10 & 1 & 3.23 & 14.59 & 0.69 & $\ldots$ & $\ldots$ & 1.05 \\
\hline HV 5551 & 1.021 & 15.00 & 0.76 & 0.86 & 0.10 & 5 & 3.21 & 14.68 & 0.66 & 0.73 & 0.65 & $\ldots$ \\
\hline HV 2432 & 1.038 & 14.23 & 0.52 & $\ldots$ & 0.10 & 1 & 3.11 & 13.92 & 0.42 & $\ldots$ & 0.74 & 0.73 \\
\hline HV 12248 & 1.038 & 14.47 & 0.75 & $\ldots$ & 0.10 & 1 & 3.21 & 14.15 & 0.65 & $\ldots$ & $\ldots$ & 1.80 \\
\hline HV 2864 & 1.041 & 14.67 & 0.80 & 0.89 & 0.07 & $1,3,4,6$ & 3.23 & 14.44 & 0.73 & 0.80 & 0.81 & 1.24 \\
\hline HV 12716 & 1.051 & 14.70 & 0.76 & $\ldots$ & 0.10 & 1 & 3.21 & 14.38 & 0.66 & 0.81 & $\ldots$ & 1.24 \\
\hline HV 2662 & 1.082 & 14.46 & 0.88 & 0.83 & 0.10 & 5 & 3.27 & 14.13 & 0.78 & 0.70 & 0.50 & $\ldots$ \\
\hline HV 2580 & 1.089 & 14.01 & 0.81 & 0.87 & 0.10 & 7 & 3.24 & 13.69 & 0.71 & 0.74 & $\ldots$ & $\ldots$ \\
\hline HV 12253 & 1.099 & 14.41 & 0.76 & $\ldots$ & 0.10 & 1 & 3.21 & 14.09 & 0.66 & 0.65 & $\ldots$ & 1.46 \\
\hline HV 874 & 1.103 & 14.45 & $\ldots$ & 0.71 & 0.10 & 5 & 7.27 & 13.72 & $\ldots$ & 0.58 & 1.10 & 1.70 \\
\hline HV 2527 & 1.112 & 14.61 & 0.83 & 0.86 & 0.10 & 1,7 & 3.24 & 14.29 & 0.73 & 0.73 & $\ldots$ & 1.52 \\
\hline HV 2260 & 1.114 & 14.85 & 0.86 & 0.96 & 0.14 & $1,3,4,7$ & 3.26 & 14.39 & 0.72 & 0.78 & $\ldots$ & 1.42 \\
\hline HV 997 & 1.119 & 14.55 & 0.86 & 0.98 & 0.11 & $1,3,4,7$ & 3.26 & 14.19 & 0.75 & 0.84 & $\ldots$ & 1.52 \\
\hline HV 2579 & 1.128 & 13.99 & 0.66 & 0.75 & 0.10 & 1,7 & 3.17 & 13.67 & 0.56 & 0.62 & $\cdots$ & 1.58 \\
\hline HV 2352 & 1.134 & 14.19 & 0.70 & 0.84 & 0.11 & $1,3,4,7$ & 3.19 & 13.84 & 0.59 & 0.70 & $\ldots$ & 1.17 \\
\hline HV 955 & 1.138 & 14.07 & 0.73 & $\ldots$ & 0.10 & 1 & 3.20 & 13.75 & 0.63 & $\ldots$ & $\ldots$ & 1.27 \\
\hline HV 2463 & 1.145 & 14.22 & 0.77 & 0.87 & 0.10 & 7 & 3.22 & 13.90 & 0.67 & 0.74 & $\ldots$ & $\ldots$ \\
\hline HV 5655 & 1.153 & 14.52 & 0.94 & 0.95 & 0.10 & 7 & 3.29 & 14.19 & 0.84 & 0.82 & $\ldots$ & $\ldots$ \\
\hline HV 2324 & 1.160 & 14.35 & 0.85 & 0.92 & 0.06 & 1,4 & 3.25 & 14.15 & 0.79 & 0.84 & $\ldots$ & 1.44 \\
\hline HV 12471 & 1.200 & 14.68 & 0.99 & $\ldots$ & 0.06 & 1,3 & 3.31 & 14.48 & 0.93 & 1.05 & 0.88 & 1.39 \\
\hline HV 2262 & 1.200 & 14.29 & 0.75 & 0.96 & 0.10 & 7 & 3.21 & 13.97 & 0.65 & 0.83 & $\ldots$ & $\ldots$ \\
\hline
\end{tabular}


Table 1. continued.

\begin{tabular}{|c|c|c|c|c|c|c|c|c|c|c|c|c|}
\hline $\begin{array}{l}\text { Cepheid } \\
\text { (1) }\end{array}$ & $\begin{array}{c}\log P \\
(2)\end{array}$ & $\begin{array}{c}V \\
(3)\end{array}$ & $\begin{array}{c}(B-V) \\
(4)\end{array}$ & $\begin{array}{c}(V-I) \\
(5)\end{array}$ & $\begin{array}{c}E(B-V) \\
(6)\end{array}$ & $\begin{array}{l}\text { Source } \\
\text { (7) }\end{array}$ & $\begin{array}{l}R_{V} \\
\text { (8) }\end{array}$ & $\begin{array}{l}V^{0} \\
(9)\end{array}$ & $\begin{array}{c}(B-V)^{0} \\
\quad(10)\end{array}$ & $\begin{array}{c}(V-I)^{0} \\
\quad(11)\end{array}$ & $\begin{array}{l}\mathcal{A}_{V} \\
(12)\end{array}$ & $\begin{array}{l}\mathcal{A}_{B} \\
\text { (13) }\end{array}$ \\
\hline HV 2549 & 1.209 & 13.70 & 0.72 & $\ldots$ & 0.06 & 1,3 & 3.19 & 13.51 & 0.66 & 0.71 & $\cdots$ & 1.63 \\
\hline HV 2580 & 1.229 & 13.96 & 0.75 & 0.86 & 0.11 & $1,3,4$ & 3.21 & 13.61 & 0.64 & 0.72 & $\ldots$ & 1.33 \\
\hline HV 2261 & 1.237 & 13.26 & 0.69 & 0.74 & 0.10 & 7 & 3.18 & 12.94 & 0.59 & 0.61 & $\ldots$ & $\ldots$ \\
\hline HV 2836 & 1.246 & 14.62 & 0.98 & 1.10 & 0.19 & $1,3,4,7$ & 3.31 & 13.99 & 0.79 & 0.86 & $\ldots$ & 1.49 \\
\hline HV 1005 & 1.272 & 13.96 & 0.82 & $\ldots$ & 0.10 & 1,4 & 3.24 & 13.64 & 0.72 & 0.84 & $\ldots$ & 1.65 \\
\hline HV 2793 & 1.283 & 14.09 & 1.01 & 1.08 & 0.10 & $1,3,4$ & 3.32 & 13.76 & 0.91 & 0.95 & $\ldots$ & 1.59 \\
\hline U 11 & 1.303 & 13.99 & 0.86 & $\ldots$ & 0.10 & 1 & 3.26 & 13.66 & 0.76 & $\ldots$ & 0.74 & 1.47 \\
\hline U 1 & 1.353 & 14.10 & 0.98 & $\ldots$ & 0.10 & 1 & 3.31 & 13.77 & 0.88 & $\ldots$ & $\ldots$ & 1.71 \\
\hline HV 2749 & 1.364 & 14.73 & 1.24 & $\ldots$ & 0.10 & 1 & 3.42 & 14.39 & 1.14 & $\ldots$ & 1.05 & 1.32 \\
\hline HV 878 & 1.367 & 13.53 & 0.67 & 0.85 & 0.06 & $1,2,3,7$ & 3.17 & 13.34 & 0.61 & 0.77 & 1.45 & 1.92 \\
\hline HV 886 & 1.380 & 13.33 & 0.83 & $\ldots$ & 0.06 & $1,2,3$ & 3.24 & 13.14 & 0.77 & 0.62 & 1.28 & 2.08 \\
\hline HV 1013 & 1.382 & 13.83 & 1.04 & 0.96 & 0.12 & $1,3,4$ & 3.34 & 13.43 & 0.92 & 0.81 & $\ldots$ & 1.49 \\
\hline HV 1003 & 1.388 & 13.25 & 0.71 & $\ldots$ & 0.06 & 1,3 & 3.19 & 13.06 & 0.65 & 0.57 & 1.05 & 1.84 \\
\hline HV 889 & 1.412 & 13.71 & 0.98 & $\ldots$ & 0.06 & $1,2,3$ & 3.31 & 13.51 & 0.92 & $\ldots$ & 0.89 & 1.66 \\
\hline HV 12815 & 1.417 & 13.48 & 0.95 & 0.99 & 0.08 & $1,3,4,6,7$ & 3.30 & 13.22 & 0.87 & 0.89 & 1.08 & 1.84 \\
\hline HV 902 & 1.421 & 13.22 & 0.70 & $\ldots$ & 0.10 & 1 & 3.19 & 12.90 & 0.60 & $\ldots$ & 1.27 & 2.10 \\
\hline HV 1023 & 1.425 & 13.75 & 0.97 & 1.03 & 0.07 & $1,3,4,7$ & 3.30 & 13.52 & 0.90 & 0.94 & $\ldots$ & 1.47 \\
\hline HV 2251 & 1.447 & 13.10 & 0.75 & $\ldots$ & 0.10 & 1,3 & 3.21 & 12.78 & 0.65 & 0.44 & 1.15 & 1.70 \\
\hline HV 2540 & 1.449 & 13.81 & 1.20 & 1.04 & 0.10 & 7 & 3.41 & 13.47 & 1.10 & 0.91 & $\ldots$ & $\ldots$ \\
\hline HV 8036 & 1.453 & 13.60 & 0.90 & $\ldots$ & 0.10 & 1,3 & 3.27 & 13.27 & 0.80 & 0.68 & $\ldots$ & 2.00 \\
\hline HV 872 & 1.475 & 13.69 & 0.96 & 0.98 & 0.10 & 7 & 3.30 & 13.36 & 0.86 & 0.85 & $\ldots$ & $\ldots$ \\
\hline HV 875 & 1.482 & 13.04 & 0.83 & 0.87 & 0.10 & $1,2,7$ & 3.24 & 12.72 & 0.73 & 0.74 & 0.45 & 1.00 \\
\hline HV 1002 & 1.484 & 12.94 & 0.76 & 0.78 & 0.00 & $1,2,3,4$ & 3.21 & 12.94 & 0.76 & 0.78 & 1.35 & 2.10 \\
\hline HV 899 & 1.492 & 13.43 & 0.94 & 1.10 & 0.11 & $1,3,4$ & 3.29 & 13.07 & 0.83 & 0.96 & 1.24 & 1.84 \\
\hline HV 882 & 1.503 & 13.42 & 0.76 & 0.98 & 0.10 & $1,2,7$ & 3.21 & 13.10 & 0.66 & 0.85 & 1.07 & 1.68 \\
\hline HV 873 & 1.536 & 13.52 & 1.09 & 1.36 & 0.10 & 1,7 & 3.36 & 13.18 & 0.99 & 1.00 & 0.71 & 1.08 \\
\hline HV 881 & 1.553 & 13.11 & 1.04 & 0.92 & 0.10 & $1,2,7$ & 3.34 & 12.78 & 0.94 & 0.79 & 1.26 & 1.95 \\
\hline HV 2294 & 1.563 & 12.66 & 0.82 & 0.95 & 0.06 & $1,2,3,4$ & 3.24 & 12.47 & 0.76 & 0.87 & 1.25 & 2.10 \\
\hline HV 879 & 1.566 & 13.35 & 1.03 & 1.06 & 0.06 & $1,3,4$ & 3.33 & 13.15 & 0.97 & 0.98 & $\ldots$ & 1.98 \\
\hline HV 909 & 1.575 & 12.75 & 0.80 & 1.01 & 0.07 & $1,2,3$ & 3.23 & 12.52 & 0.73 & 0.92 & 0.92 & 1.45 \\
\hline HV 2257 & 1.592 & 13.04 & 0.96 & 1.04 & 0.06 & $1,2,3,4$ & 3.30 & 12.84 & 0.90 & 0.96 & 1.30 & 2.06 \\
\hline HV 2338 & 1.625 & 12.78 & 0.94 & 0.95 & 0.07 & $1,3,4$ & 3.29 & 12.55 & 0.87 & 0.86 & 1.14 & 1.94 \\
\hline HV 877 & 1.655 & 13.35 & 1.20 & 1.19 & 0.11 & $1,2,3,4$ & 3.41 & 12.98 & 1.09 & 1.05 & 0.69 & 1.01 \\
\hline HV 900 & 1.677 & 12.78 & 0.92 & 0.92 & 0.09 & 1,3 & 3.28 & 12.48 & 0.83 & 0.80 & 1.08 & 1.74 \\
\hline HV 953 & 1.680 & 12.28 & 0.87 & 0.90 & 0.09 & $1,2,3,4$ & 3.26 & 11.99 & 0.78 & 0.78 & 0.95 & 1.60 \\
\hline HV 2369 & 1.684 & 12.61 & 0.96 & 1.02 & 0.10 & $1,3,4$ & 3.30 & 12.28 & 0.86 & 0.89 & 1.13 & 1.75 \\
\hline HV 270100 & 1.872 & 11.79 & 0.96 & $\ldots$ & 0.06 & 3 & 3.30 & 11.59 & 0.90 & $\ldots$ & $\ldots$ & $\ldots$ \\
\hline HV 2827 & 1.897 & 12.30 & 1.24 & 1.08 & 0.08 & $1,3,4$ & 3.42 & 12.03 & 1.16 & 0.98 & $\ldots$ & 0.94 \\
\hline HV 5497 & 1.995 & 11.93 & 1.20 & 1.13 & 0.09 & $1,2,3,4$ & 3.41 & 11.62 & 1.11 & 1.01 & 0.56 & 0.95 \\
\hline HV 2883 & 2.033 & 12.41 & 1.23 & 1.08 & 0.01 & $1,2,4$ & 3.42 & 12.38 & 1.22 & 1.07 & 1.17 & 1.92 \\
\hline HV 2447 & 2.077 & 12.00 & 1.25 & 1.11 & 0.04 & $1,2,4$ & 3.43 & 11.86 & 1.21 & 1.06 & 0.57 & 0.89 \\
\hline HV 883 & 2.127 & 12.14 & 1.19 & 1.10 & 0.09 & $1,2,4$ & 3.40 & 11.83 & 1.10 & 0.98 & 1.23 & 1.80 \\
\hline
\end{tabular}

Sources: (1) Martin et al. (1979), (2) Madore (1985), (3) Laney \& Stobie (1994), (4) Caldwell \& Coulson (1985), (5) Martin (1980),

(6) Caldwell et al. (1986), (7) Sebo et al. (2002).

Cepheids from Paper I. The overall slope of the LMC P-C relations (Eqs. (1) and (4)) are similar to the Galactic P-C relations in $(B-V)^{0}$ and $(V-I)^{0}$, but the best-fit LMC slopes, which are different for Cepheids with $\log P \gtrless 1.0$, are significantly flatter for short-period and steeper for long-period Cepheids. In addition the bulk of the LMC Cepheids is considerably bluer in $(B-V)^{0}$ and $(V-I)^{0}$ than the Galactic Cepheids. The mean color difference $\Delta(B-V)_{\text {Gal-LMC }}^{0}$ amounts to 0.05 at $\log P=0.4$, to increase to $\sim 0^{\mathrm{m}} 10$ at $\log P=1.0$, and to decrease to 0.04 at $\log P=1.8$. The corresponding, smaller color differences $\Delta(V-I)_{\text {Gal-LMC }}^{0}$ are $0.03, \sim 0.08$, and $0 .{ }^{\mathrm{m}} 01$.
The color differences between LMC and Galactic Cepheids must be due to blanketing and/or temperature differences. This point will be followed up in Sect. 3.4.

\subsection{The two-color diagram of $L M C$ and Galactic Cepheids}

The mean position of the LMC Cepheids in a plot of $(B-$ $V)^{0}$ vs. $(V-I)^{0}$ is shown with two full lines for $\log P \gtrless$ 1.0 in Fig. 2. The lines are determined from a combination of Eqs. (2), (5) and (3), (6), respectively. Also shown in the 
Table 2. The color and magnitude differences between the U99 and Table 1 samples in the sense $\Delta=\Delta_{\mathrm{U} 99}-\Delta_{\text {Table } 1}$.

\begin{tabular}{cccccc}
\hline \hline & $(B-V)^{0}$ & $(V-I)^{0}$ & $B^{0}$ & $V^{0}$ & $I^{0}$ \\
\hline$\Delta$ & -0.008 & -0.015 & +0.017 & +0.013 & +0.009 \\
$\epsilon$ & \pm 0.012 & \pm 00.013 & \pm 000.041 & \pm 0.032 & \pm 00.035 \\
\hline
\end{tabular}

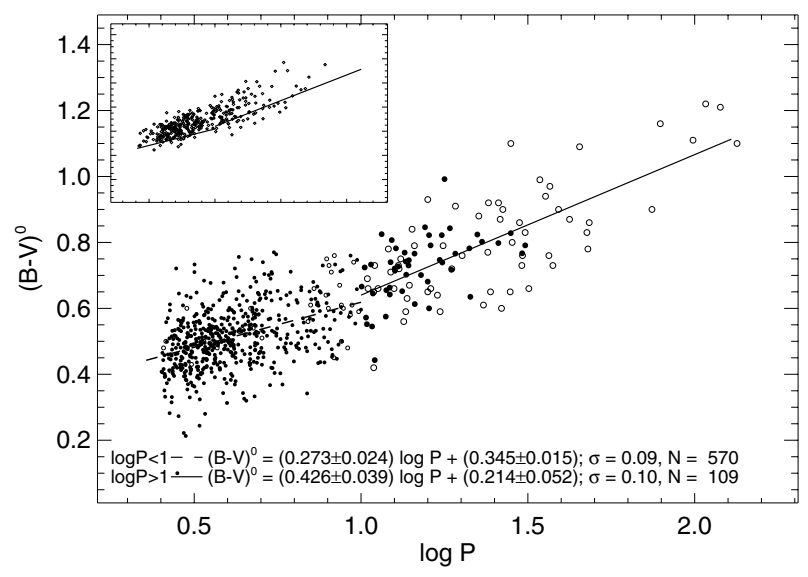

Fig. 1a. The P-C relation in $(B-V)^{0}$ of 679 LMC Cepheids. Black dots are from U99; small dots for $\log P<1.0$, large dots for $\log P>$ 1.0. Open symbols are from Table 1. Two separate fits for Cepheids with $\log P \gtrless 1.0$ are shown. The insert repeats the mean LMC relations and shows the individual Galactic Cepheids from Paper I.

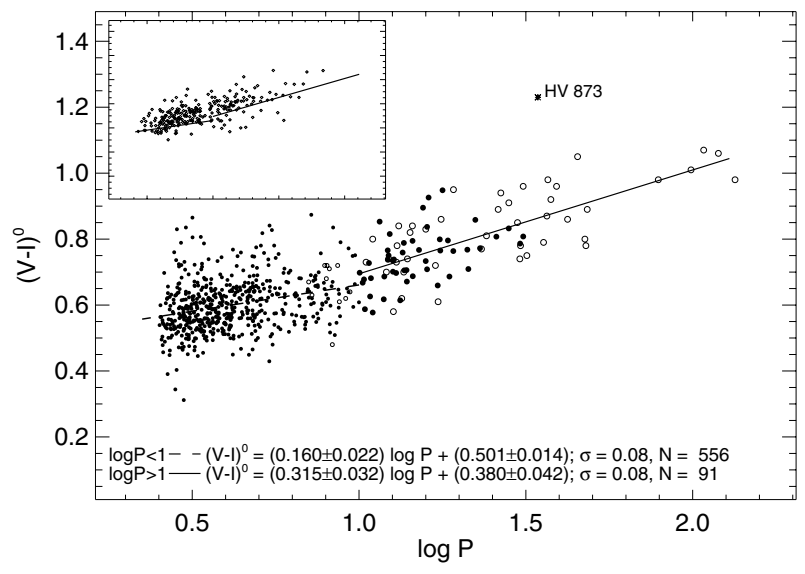

Fig. 1b. The P-C relation in $(V-I)^{0}$ of 634 LMC Cepheids. Symbols and insert as in Fig. 1a. Separate linear regressions are shown for Cepheids with $\log P \gtrless 1.0$.

figure is the mean position of Galactic Cepheids (dashed line), where the position of the line and of the different $\log P$ values is taken from Paper I.

Figure 2 is the comparison of the two color diagrams of the Galaxy and LMC. It differs subtly from the similar diagram $7 \mathrm{~b}$ in Paper I where the LMC relation is slightly above that for the Galaxy, whereas in Fig. 2 here it is below the Galaxy line for $\log P>1,(B-V)^{0}>0.65$. The difference is that the $\mathrm{P}-\mathrm{C}$ relations (Figs. 3 and 5 in Paper I; Figs. 1a and 1b here) were fit in Paper I by a straight line, whereas here the break at $\log P=1$ is allowed for. This now lets LMC Cepheids with $\log P>1$

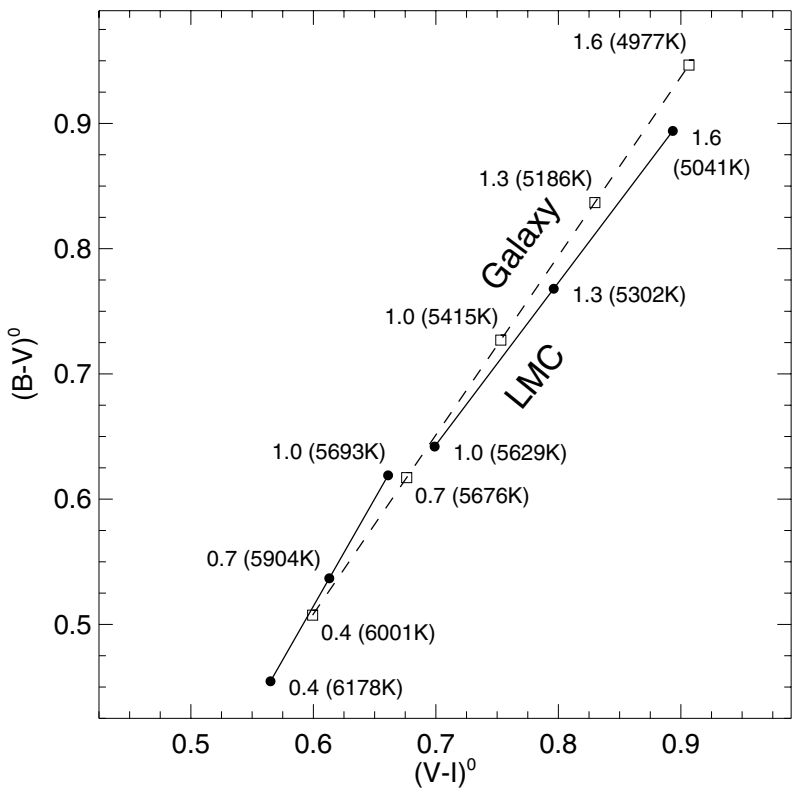

Fig. 2. The two color diagram of Cepheids in LMC (full line) and the Galaxy (dashed). Loci of different values of $\log P$ are labelled as well as the corresponding temperature $T_{\mathrm{e}}$.

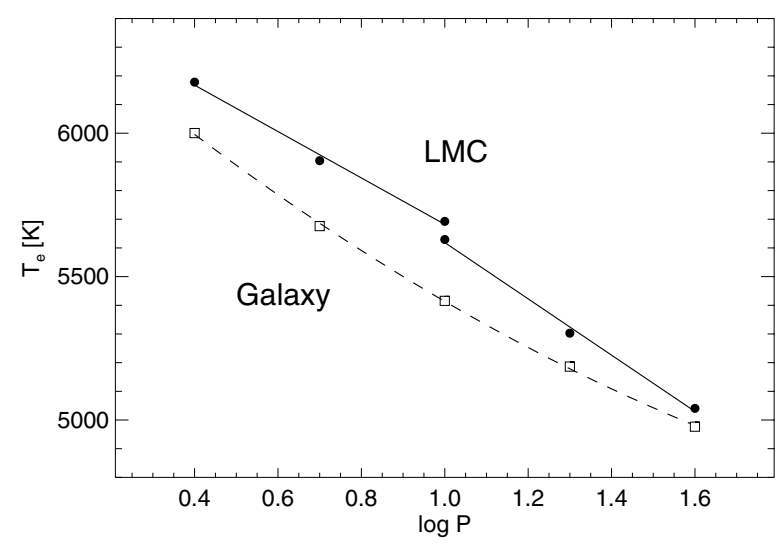

Fig. 3. The $\log P-T_{\mathrm{e}}$ variation of LMC and Galactic Cepheids.

be redder in $(V-I)^{0}$ than Galactic ones for $(B-V)^{0}>0.65$ as the models of Bell and Tripicco (Sandage et al. 1999, hereafter SBT) require due to the lower metallicity of LMC (cf. Fig. 7a panel d of Paper I).

However, a comparison of the mean relations in Fig. 2 does not tell the full story. The points of equal period are shifted to each other. LMC Cepheids of given period are bluer in $(B-V)^{0}$ by $0^{\mathrm{m}} .05-0.0^{\mathrm{m}} 10$ and in $(V-I)^{0}$ by roughly $0^{\mathrm{m}} 05$ than their Galactic counterparts. The reason is as follows.

There is a real temperature difference (see Sect. 8) in the ridge-line $M_{V}, \log T_{\mathrm{e}}$ diagram (the HR diagram of Fig. 20, later) for the Galaxy compared with LMC. The calculations set out later in Sect. 8 lead to Fig. 3, showing an average temperature difference of about $150^{\circ}$, ranging from $80 \mathrm{~K}$ to $350 \mathrm{~K}$ depending on the period. Although our mean value is smaller than the $250^{\circ}$ found by Laney \& Stobie (1986) using $(J-K)$ colors, it is in the same sense. 
It is clear why this temperature shift toward the blue for LMC causes the period separations at given colors between LMC and the Galaxy in Fig. 2 (and Fig. 7b in Paper I). The lines of constant period that thread the instability strip (Fig. 9 later) cut a hotter central (ridge-line) position at bluer color in LMC than for the a cooler ridge-line position in the Galaxy. Hence, for LMC, the $(B-V)^{0}$ and $(V-I)^{0}$ colors at a given period are bluer than for the Galaxy at the same period. This is the sense of the scrambling of periods in Fig. $7 \mathrm{~b}$ of Paper I and Fig. 2 here.

Possible reasons for the shift in the temperatures of the ridge-line HRDs between the Galaxy and LMC are discussed in Sect. 8.

\section{The Period-Luminosity relations of LMC}

\subsection{The $P-L$ relation of $L M C$ in $B, V$, and I}

It would be possible to discuss the P-L relation of LMC using the dereddened apparent magnitudes only. We prefer, however, to use absolute magnitudes which allow also a comparison with the Galactic zero points. We adopt $(m-M)_{\mathrm{LMC}}^{0}=18.54$ as the best Cepheid-independent distance modulus as justified in Paper I. This choice has no qualitative consequences for the following conclusions, and the reader can adjust the quantitative results to any preferred distance of LMC.

The absolute magnitudes $M_{B V I}^{0}$ of the sample Cepheids follow directly from the adopted LMC modulus and the color excesses discussed in Sect. 2.3. The adopted absorption law follows in Sect. 4.2.1. The resulting absolute magnitudes are plotted against $\log P$ in Fig. 4. If the data in the three panels of Fig. 4 are fit over the entire period range by linear regressions, one obtains $(N=679,689,647)$

$M_{B}^{0}=-(2.340 \pm 0.037) \log P-(1.200 \pm 0.029), \sigma=0.29,(7)$

$M_{V}^{0}=-(2.702 \pm 0.028) \log P-(1.491 \pm 0.022), \sigma=0.22,(8)$

$M_{I}^{0}=-(2.949 \pm 0.020) \log P-(1.936 \pm 0.015), \sigma=0.14$. (9)

These fits,which are in reasonable agreement with previous P-L relations of LMC (e.g. Kraft 1961; Sandage \& Tammann 1968; Laney \& Stobie 1994; Gieren et al. 1998) and close to the solutions in $V$ and $I$ by Udalski et al. (1999a), systematically overestimate the luminosity of Cepheids around $\log P=1.0$, and are hence unsatisfactory. A better fit is obtained when the P-L relations in $B, V$, and $I$ are each fit with two linear regressions breaking at $\log P=1.0$. The two-slopes solutions are shown in Fig. 4. The significance of the slope differences between short- and long-period Cepheids varies between 2.6 $\sigma(I)$ and $3.1 \sigma(V)$. The reality of the break is supported by the broken P-C relations in Sects. 3.1 and 3.2 since any non-linearity of the $\mathrm{P}-\mathrm{C}$ relations must be reflected in the $\mathrm{P}-\mathrm{L}$ relations.

Least-squares solutions to the data in Fig. 4 with two linear regressions, breaking at $\log P=1.0$, give in $M_{B}$ and for

$\log P<1(N=570)$ :

$M_{B}^{0}=-(2.683 \pm 0.077) \log P-(0.995 \pm 0.049), \sigma=0.27,(10)$

$\log P>1(N=109)$ :

$M_{B}^{0}=-(2.151 \pm 0.134) \log P-(1.404 \pm 0.177), \sigma=0.35,(11)$ in $M_{V}$ and for

$\log P<1(N=579)$ :

$M_{V}^{0}=-(2.963 \pm 0.056) \log P-(1.335 \pm 0.036), \sigma=0.20$,

$\log P>1(N=110)$ :

$M_{V}^{0}=-(2.567 \pm 0.102) \log P-(1.634 \pm 0.135), \sigma=0.27$,

and in $M_{I}$ and for

$\log P<1(N=556):$

$M_{I}^{0}=-(3.099 \pm 0.038) \log P-(1.846 \pm 0.024), \sigma=0.13$,

$\log P>1(N=91)$ :

$M_{I}^{0}=-(2.822 \pm 0.084) \log P-(2.084 \pm 0.111), \sigma=0.21$.

The luminosity scatter of the long-period Cepheids is larger than at periods less than 10 days. This could be due to a broadening of the instability strip in the CMD at longer periods or to steeper constant-period lines (see Sect. 6.2 below). But it could also be due to the Cepheids in Table 1 which carry much of the weight at longer periods. Their extinctions $E(B-V)$ are less uniformly determined than the U99 sample, and, scattered over the face of LMC, they may be more affected by the depth effect of the galaxy than the U99 Cepheids which lie along the bar of LMC. It is therefore not possible to identify a single reason for the larger scatter of long-period Cepheids.

\subsection{Comparison with the Galaxy}

\subsubsection{A revision to the Galactic P-L relations}

In Paper I we have derived the Galactic P-L relation in $B, V$, and $I$ from the then available 25 fundamental-mode Cepheids in clusters and associations from Feast (1999) and 28 Cepheids with Baade-Becker-Wesselink (BBW) expansion parallaxes from Gieren et al. (1998). In the meantime additional distances of cluster/association Cepheids have become available (Turner \& Burke 2002; Hoyle et al. 2003). Overlapping cluster distances of the three sources show no systematic difference after they have been reduced to a common zero point. The zero point was based on the Pleiades at $(m-M)^{0}=5.61$, a value which was determined from careful photometry by Stello \& Nissen (2001). This value agrees within \pm 0 m 05 with the photometric distance of Pinsonneault et al. (1998), the distance of the double star Atlas (Pan et al. 2004), the moving-cluster distance (Narayanan \& Gould 1999), the rotation period method of latetype members (O'Dell et al. 1994), as well as now also with the latest HIPPARCOS parallax (Makarov 2002). Distances are now known for 33 Cepheids which are members of clusters or associations (the deviating CGCas and AQPup are excluded as too uncertain cluster members). In cases where more than one independent distance determination is available weighted means were adopted.

The BBW distances of Gieren et al. (1998) have been superceded by the revised distances of Fouqué et al. (2003) who have also added four new Cepheids. We have augmented their list by the BBW distances of Barnes et al. (2003). Also included are the distances from interferometric diameter observations of $\delta$ Cep (Benedict et al. 2002; Nordgren et al. 2002), 


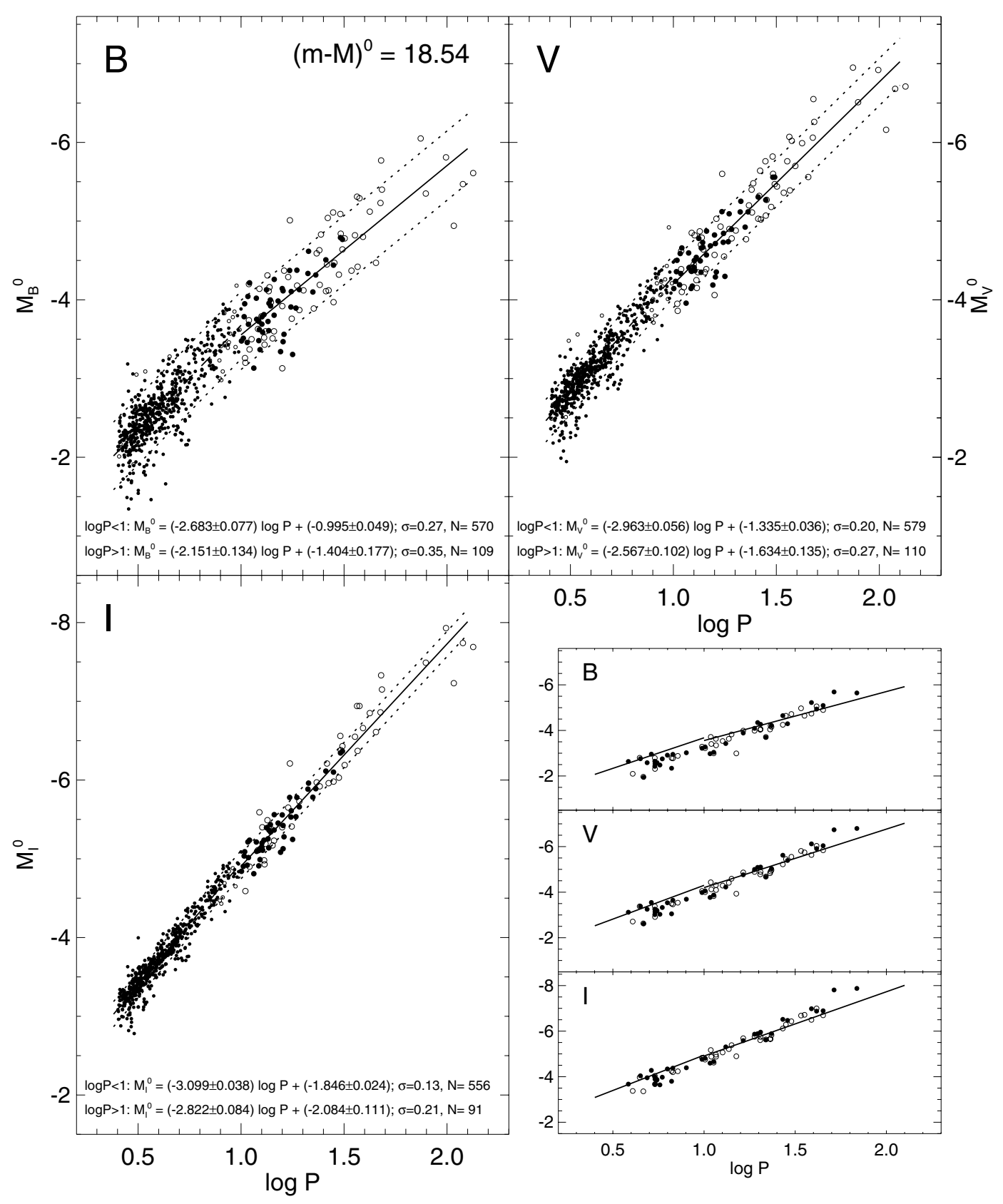

Fig. 4. The P-L relations in $B, V$, and $I$ of LMC Cepheids. The data in each color are fitted with two linear regressions breaking at log $P=1.0$. Symbols as in Fig. 1a. For the dashed intrinsic boundaries see text (6.2). Comparison with the revised Galactic calibration in Sect. 4.2.1 (Eqs. (16)-(18)) are in the lower right panel. The individual Galactic Cepheids with known absolute magnitudes (cf. Sect. 4.2.1) are the data points. The LMC mean relations are the solid lines.

$\eta$ Aql (Lane et al. 2002; Kervella et al. 2004), $\zeta$ Gem (Lane et al. 2002), and 1 Car (Kervella et al. 2004). Unweighted means have been adopted for Cepheids with more than one BBW distance. This brings the total of BBW distances to 36 Cepheids.

Kovács (2003) has published independent BBW absolute magnitudes of 25 Galactic Cepheids. His results for $\log P>1$ are in excellent agreement on average with the above data, yet his magnitudes for $\log P<1$ are $\sim 0$. 5 brighter on average than the latter. Correspondingly his suggested slope of the Galactic P-L relation is much flatter than from either Fouqués et al. results or the cluster Cepheids. For this reason his results are not used here.
The Galactic P-L relations in $B, V$, and $I$ as defined by the 33 cluster Cepheids ${ }^{3}$ and the 36 Cepheids with BBW distances are shown in Fig. 5. Separate linear regressions of either set are given at the bottom of each panel in Fig. 5. The agreement of the P-L relations from entirely independent distance determinations is impressive. The cluster data give marginally steeper slopes. If the BBW distances of the longest-period Cepheids GY Sgr and V Vul had been included from Gieren et al. (1998) the agreement in slope would be nearly perfect;

${ }^{3}$ I-magnitudes of new calibrators not in Table 1 of Paper I are taken from Lanoix et al. (1999). No I-magnitude is available for TV CMa. 


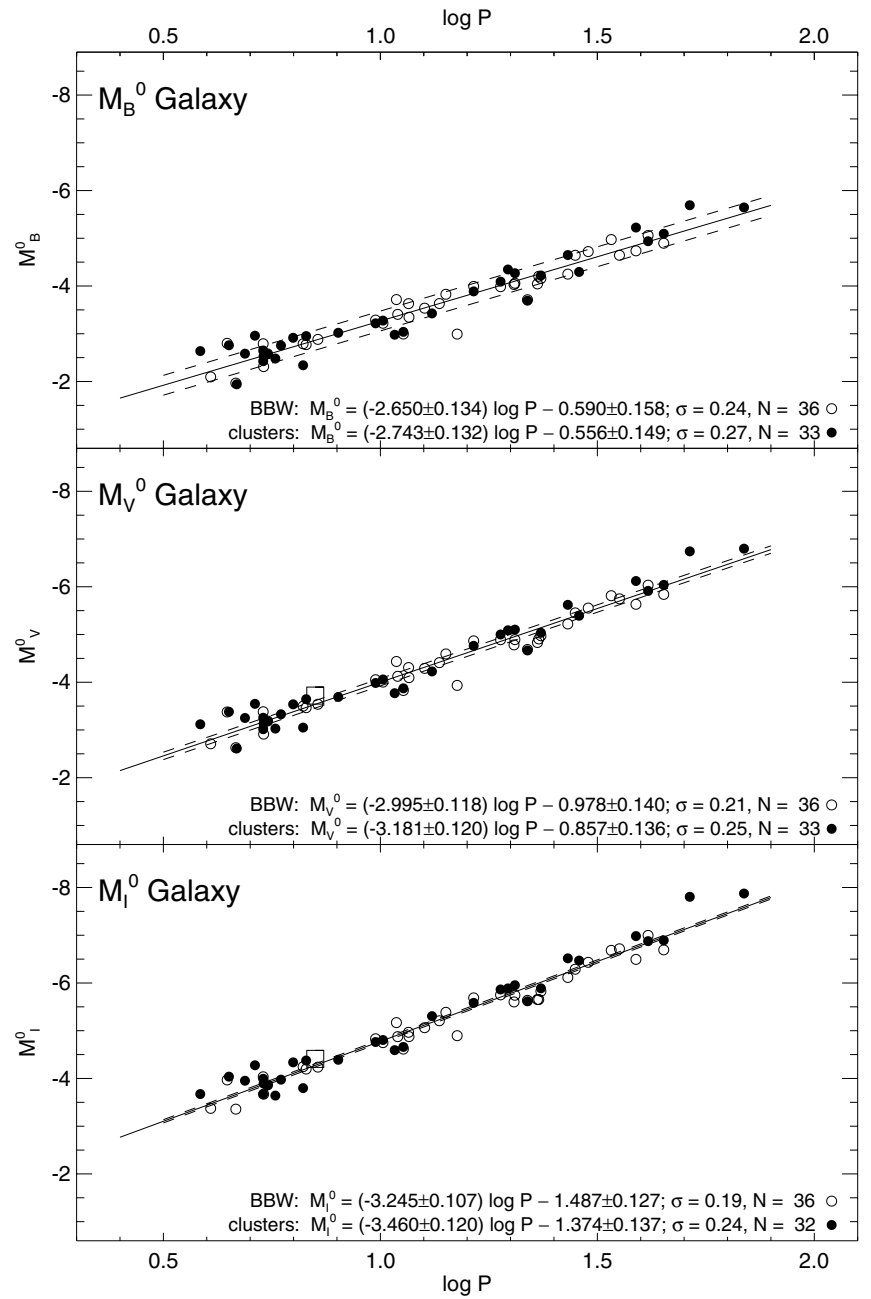

Fig. 5. The Galactic P-L relations in $B, V$, and $I$ from 36 Cepheids with cluster distances and 33 (32) Cepheids with BBW distances. The mean relations from Eqs. (16)-(18) are shown as full lines. The open square is from the HIPPARCOS calibration by Groenewegen \& Oudmaijer (2000). The dashed boundaries are explained in the text.

however they have been excluded by Fouqué et al. (2003) because of their variable period which impairs the match between photometric and radial-velocity data. - The independent pairs of $\mathrm{P}-\mathrm{L}$ relations in $B, V$, and $I$ agree to within 0.01 at $\log P=0.5$. They diverge by up to $0.11,0.14$, and 0.21 in the three colors at long periods $(\log P=1.5)$, the cluster data being brighter.

One may suspect that the (small) slope difference is rather due to errors of the BBW distances than of the cluster distances, because it is hard to explain why the latter should depend on the period of a Cepheid. However, since it is impossible to objectively judge the systematic errors which affect the two independent methods we have joined the cluster and BBW Cepheids with equal weights, which leads to the following combined P-L relations for our Galaxy $(N=$ $69,69,68)$ :

$M_{B}^{0}=-(2.692 \pm 0.093) \log P-(0.575 \pm 0.107), \sigma=0.25(16)$

$M_{V}^{0}=-(3.087 \pm 0.085) \log P-(0.914 \pm 0.098), \sigma=0.23(17)$

$M_{I}^{0}=-(3.348 \pm 0.083) \log P-(1.429 \pm 0.097), \sigma=0.23 .(18)$
The main difference of these revised Galactic P-L relations with those derived in Paper I is that they are based now on a significantly broader base with correspondingly smaller statistical errors of the coefficients and even marginally reduced scatter. The new relations are brighter in $B, V$, and $I$ for all periods, but nowhere by more than 0.07 . They agree exceedingly well with the Galactic P-L relations of Ngeow \& Kanbur (2004) and Storm et al. (2004) which are derived on somewhat different precepts. The deviations in $B, V$, and $I$ are never larger than 0 . 07 over the entire interval of $0.4<\log P<1.5$.

As discussed in Paper I the HIPPARCOS zero point in $V$ and $I$ by Groenewegen \& Oudmaijer (2000) is somewhat brighter than the combined cluster and BBW calibration, but the difference is now reduced to $\Delta V=0.18 \pm 0.11$ and $\Delta I=0 . \mathrm{m}^{\mathrm{m}} 14 \pm 0.12$.

Also the HST parallax (Benedict et al. 2002) of $\delta$ Cep $(\log P=0.730)$ is rather bright with $M_{V}=-3.47 \pm 0.10$ (or -3.41 according to Feast 2002), while the P-L relation of Eq. (17) predicts $M_{V}=-3.17 \pm 0.10$ and the Galactic P-L-C relation as given in Table 4 (below) $M_{V}=-3.19 \pm 0.10$. The difference amounts to $\sim 0.25 \pm 0.14$. The agreement is somewhat better with the HIPPARCOS zeropoint of Feast \& Catchpole (1997), giving $M_{V}=-3.55 \pm 0.10$ at a median period of $\langle\log P\rangle=0.80$, which is to be compared with $M_{V}=$ $-3.38 \pm 0.10$ from Eq. (17), and also with the zeropoint of Lanoix et al. (1999) of $M_{V}=-3.59 \pm 0.10$ at $\langle\log P\rangle=0.82$ instead of $M_{V}=-3.45 \pm 0.10$ here. (The values of Feast \& Catchpole and Lanoix et al. are here reduced to the $E(B-V)_{\text {corr }}$ as described in Paper I). Thus, considering the parallax data one could conclude that the present zeropoint was too faint by $\sim 0.15$ in $V,-$ a possibility which on statistical grounds cannot be excluded. It should be noted, however, that the BBW and cluster distances agree formally to within 0.04 at the relevant periods of $\langle\log P\rangle \approx 0.80$.

The absorption-to-reddening ratios $\mathcal{R}_{B V I}$ were derived in Paper I from the then available Cepheid calibrators by requiring that their residuals from the mean P-L relations be not a function of $E(B-V)$. The now available 69 (68) calibrators give slightly revised values, i.e.

$\mathcal{R}_{B}=4.23, \quad \mathcal{R}_{V}=3.23, \quad \mathcal{R}_{I}=1.95$.

To be exact these values apply for Cepheids with median color of $(B-V)=0.766$ and median color excess $E(B-V)=0.382$. The dependence of $\mathcal{R}_{B V I}$ on Cepheid color and $E(B-V)$ remains the same as in Eq. (8) in Paper I.

In the remainder of this paper Eqs. (16)-(18) are referred to as the (revised) "Galactic P-L relations". The adopted P-C relations of Paper I remain unaffected by this revision.

The new Galactic P-L relation in $V$ gives in combination with the Galactic P-C relations in Paper I (Eqs. (3) and (5)) the ridge line equations of the instability strip in the CMD. One obtains (in revision of Paper I):

$M_{V}=-(8.43 \pm 0.41)(B-V)+2.13 \pm 0.21$

$M_{V}=-(12.04 \pm 0.86)(V-I)+5.08 \pm 0.48$.

In Paper I the slope $\beta$ of the constant-period lines in the CMD was not determined. With the increased sample of 
Galactic calibrators this is attempted here. The result for the $M_{V}-(B-V)$ plane, averaging over all periods, is $\beta_{V, B-V}=$ $0.60 \pm 0.40$ (see Table 4 below). This slope has still a considerable error, but it is significantly flatter than the canonical semitheoretical value of $\beta_{V, B-V} \approx 2.5$ (Sandage \& Tammann 1969; Sandage 1972). Also the slope of the constant-period lines in the $M_{V}-(V-I)$ plane is quite flat, i.e. $\beta_{V, V-I}=0.66 \pm 0.41$ (Table 4). Since the slope in the $M_{I}-(V-I)$ plane is one unit smaller (see Eq. (44)) it becomes negative, i.e. $\beta_{I, V-I}=-0.34$. Cepheids near the red edge of the instability strip are therefore (slightly) brighter in $I$ than their bluer counterparts.

The flat constant-period lines of Galactic Cepheids have an interesting consequence in as much as the intrinsic width of the Galactic P-L relation becomes quite narrow. The width is given by the product of the width of the instability strip and of the slope of the constant-period lines. The strip width was found to be $\Delta(B-V)= \pm 0.13$ and $\Delta(V-I)= \pm 0.10$ in Paper I from only 53 Galactic calibrators with independently known absolute magnitudes, but they are supported by the 16 additional calibrators considered here. Multiplying the strip width with the appropriate constant-period line slopes $\left(\beta_{B, B-V}=1.60, \beta_{V, B-V}=0.60, \beta_{I, V-I}=-0.34\right)$ gives predicted half-widths of the P-L relation of $\pm 0.21, \pm 0.08$, and \pm 0.03 in $B, V$, and $I$. The corresponding boundaries of the P-L relation in $B, V$, and $I$ are shown in Fig. 5 as dashed lines. The tight P-L relations found here for the Galaxy with its relatively high metallicity are opposite to Allen \& Shanks (2004) who have suggested that the intrinsic dispersion increased with the metallicity.

The expected narrow boundaries of the P-L relations in Fig. 5 are seemingly in conflict with the individual Cepheids defining the mean relations, but their scatter is mainly caused by the random errors of the cluster and BBW distances and is therefore about equal in $B, V$, and $I$ (see Eqs. (16)-(18)).

\subsubsection{Comparisons of the Galaxy and LMC P-L relations}

A comparison between the $\mathrm{P}-\mathrm{L}_{B V I}$ relations in $\mathrm{LMC}$ and in the Galaxy is shown in the lower right panel of Fig. 4, where the mean P-L relations of LMC are repeated together with the individual Galactic Cepheids with known absolute magnitudes from Sect. 4.2.1. It is obvious that the LMC relations above 10 days are much flatter than in the Galaxy. This causes the luminosity difference between LMC and Galactic Cepheids to be a function of period. At $\log P=0.4 \mathrm{LMC}$ Cepheids are brighter by 0.42 to $0^{\mathrm{m}} .32$ in $B, V$, and $I$ than their Galactic counterparts. The luminosity difference decreases towards longer periods and vanishes at $\log P=1.5$ in $B, \log P=1.4$ in $V$, and $\log P=1.25$ in $I$. At still larger periods Galactic Cepheids are more luminous. The hypothesis that the P-L slopes of the Galaxy and LMC are the same is rejected by a t-test with a probability of more than 95\% (Ngeow \& Kanbur 2004).

The practical consequence of this astonishing result is that it is in principle impossible to derive a reliable Cepheid distance of LMC by means of a Galactic P-L relation. Any derived distance depends necessarily on the period distribution of the
Cepheids under consideration. Even the above statement, that the LMC and Galactic Cepheids have equal luminosities somewhere near $\log P \approx 1.4$, offers no consolation because it depends entirely on the adopted distance of $(m-M)_{\mathrm{LMC}}=18.54$.

It was shown in Paper I that at least part of the observed differences of slope of the P-L relations of the Galaxy and LMC is a necessary consequence of metallicity differences. We will return to this point in Sect. 7 .

\subsection{Comparison with model calculations}

The model P-L relations of LMC in $M_{V}$ of Baraffe \& Alibert (2001) and - in their linear solution - of Caputo et al. (2000), based on independent stellar evolution and pulsation codes, agree well with the observed relations in Eqs. (12), (13) and Fig. $4 \mathrm{~b}$, yet they do not predict the break at $\log P=1$. In spite of this the difference between theoretical and observed mean magnitudes is nowhere larger than 0.2 over the entire interval $0.4<\log P<1.5$. The same statement holds for the additional $M_{I}$ magnitudes by Caputo et al. (2000). Thus their $M_{V}$ and $M_{I}$ magnitudes fit the LMC observations much better than in the case of the Galaxy (cf. Paper I).

However, Baraffe \& Alibert's (2001) $M_{B}$ magnitudes are too faint by $\sim 0$. 3 for shorter-period Cepheids, while those of Caputo's et al. (2000) quadratic solution are too faint by the same amount at short and long periods and by less at intermediate periods. The faintness of the predicted theoretical $M_{B}$ magnitudes causes the LMC Cepheids to appear too red at the corresponding periods.

The steep $\mathrm{P}-\mathrm{L}_{B V I}$ relations derived from various evolution and pulsation codes by SBT refer to the blue edge of the instability strip. No attempt was made to reduce them to the mean relations because additional assumptions would be needed.

The only theoretical $\mathrm{P}-\mathrm{L}$ relations which predict a flattening above $\log P=1$ are the quadratic solutions by Caputo et al. (2000), although they predict a gradual flattening instead of $a$ break, which leads to too faint magnitudes at very long periods.

\section{The significance of the break at $\log P=1$}

A gradual change of slope of the Cepheid P-L relation with increasing period was suggested several times (e.g. Sandage \& Tammann 1968; Sandage 1972; SBT; Caputo et al. 2000), but a sudden change of slope of the P-C and P-L relations of LMC, as already suggested by Tammann et al. (2002) and Tammann $\&$ Reindl (2002), came as a surprise. In Sects. 3 and 4 we have re-analyzed the situation and found that the break is statistically significant. The significance of the break becomes overwhelming from the shape of the LMC instability strip in the CMD (see Sect. 6.1 later). Since the break has not been predicted by any models, and since it has not been found in the Galaxy - perhaps only because of the fewer number of Galactic Cepheids with known absolute magnitudes - it may still appear as an ad hoc result.

Although we cannot offer a physical explanation for the break, we compile in the following evidence that several Cepheid parameters undergo a rather abrupt change at $\log P=1$. 


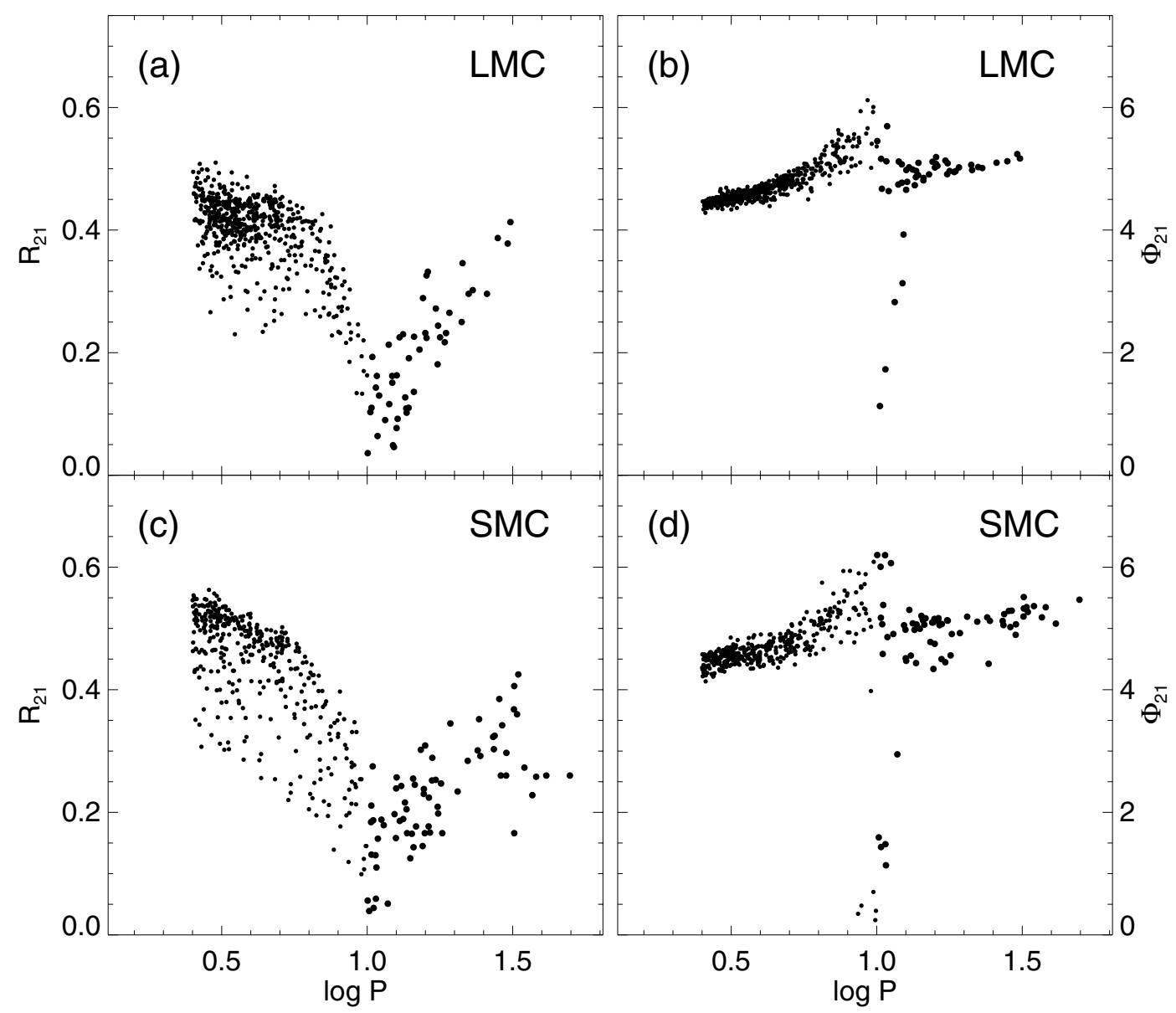

Fig. 6. The Fourier coefficients $R_{21}$ and $\Phi_{21}$ in function of $\log P$ for LMC (upper panel) and SMC (lower panel) Cepheids. In spite of some systematic differences in $R_{21}$, the minimum of $R_{21}$ and $\Phi_{21}$ occurs in either galaxy at $\log P=1$. Small and large symbols are for $\log P \gtrless 1$.

(1) In Fig. 6 the Fourier coefficients $R_{21}=A_{2} / A_{1}$ and $\Phi_{21}=$ $\Phi_{2}-2 \Phi_{1}$ of the $I$-band light curves of LMC and SMC, as given by Udalski et al. (1999b,c), are plotted versus $\log P$ (cf. also Fig. 3 of Udalski et al. 1999b). Here $A_{i}$ and $\Phi_{i}$ are the amplitude and phase of the $(i-1)$ th harmonic (cf. Simon \& Lee 1981). The diagrams, both for $R_{21}$ and $\Phi_{21}$, show a striking discontinuity close to $\log P=1$. This is the period where the hump of the well known Ludendorff-Hertzsprung progression (Ludendorff 1919; Hertzsprung 1924, 1926) reaches the phase of maximum light, and from that period and longer the hump appears on the ascending branch of the light curve. (Note: in calculating the $\Phi_{21}$ values from the listed Fourier coefficients by Simon $\&$ Lee one must add progressive increments of $2 \pi$ until one gets a positive number, in order to reproduce the discussion by Simon \& Lee. Even so, they have added one extra $2 \pi$ increment to Cepheids near $\log P=1$ to produce an uprising $\Phi_{21}$ curve near $\log P=1$ instead of the downward progression we show here, using one factor of $2 \pi$ smaller.)

It is now useful to take a side excursion to discuss a few properties of the Fourier components. What do they mean?

Schaltenbrand \& Tammann (1971) introduced Fourier series to describe the light curves of Galactic Cepheids simply as an objective way to derive many properties of the curves such as the amplitude, phase of maximum light, and fraction of the period between minimum and maximum light. They did not list the Fourier coefficients because these authors used them only as an intermediate step to derive accurate values of these observed parameters that are manifest from the observations.

On the other hand, Simon \& Lee (1981) needed an objective way characterize light curve shapes to make easier comparisons with Simon's (and a multitude of others; see Simon \& Lee for a summary to 1981) hydrodynamic calculations of theoretical light curves, circumventing the need to describe in words the "bumps", "shoulders" and "standstills" in fitting the calculations with the observations. The comparisons between theory and the observations, argued Simon and Lee, would best be made by deriving selected Fourier coefficients from the observed light curves, and comparing them with the Fourier decompositions of theoretical light curves. This was the reason for the initial work by Simon and Lee in decomposing the observations of Galactic Cepheids in Fourier series.

Yet the $R_{21}$ and $\Phi_{21}$ values shown in Fig. 6, beg for some description useful for observers of what is actually seen by visual inspection of the light curves. However, because higher order terms are not used for $R_{21}$ or $\Phi_{21}$, we cannot expect an exact description of light curve shapes using only the first two terms. Nevertheless, a few conclusions concerning the correspondence with the observations are evident, especially for $R_{21}$.

Clearly, if a light curve is a perfect sine or cosine curve, all $R_{i}$ in a Fourier series will be zero for all $i>2$. 
Therefore, $R_{21}=0$ in that case. It is also easily shown (any text on Fourier analysis) that all symmetrical curves with finite first derivatives (no cusps), where the rise from minimum to maximum is half the period, also have $R_{21}$ near 0 . Hence, small $R_{21}$ means more symmetrical light curves than large $R_{21}$ values. This is precisely the first fact of the Ludendorff-Hertzsprung progression of light curve shapes.

Hertzsprung (1924) writes: "it has been pointed out by Ludendorff - that for [Cepheid] variable stars [those] of periods between one and two weeks have the most symmetrical lightcurves". This is shown decisively by Ludendorff's Fig. 1 where the rise times of 91 Cepheids with periods greater than 1 day show nearly symmetrical (rise times $=0.5$ phase units) light curves from 7 to 12 days, whereas the light curve shapes for longer and shorter period are much more asymmetrical (high $R_{21}$ values). It is this fact that is evident in the dip where $R_{21}$ approaches zero near 10 days in Fig. 6.

We can also understand why the maximum value of $R_{21}$ is approximately 0.5 for shorter periods. At these periods, the light curves are most highly peaked (i.e. very asymmetrical). In the limit of large "peakedness", the curves can be approximated by a saw tooth with very short rise times (actually zero for a pure sawtooth with a vertical rise at 0 and $2 \pi$ phases). It is well known that for this extreme saw tooth, the first term in a Fourier series is $\sin x$ and the second term is $0.5 \sin 2 x$. Hence $R_{1}=1$ and $R_{2}=0.5$. Hence, $R_{21}=R_{2} / R_{1}=0.5$ by the definition of $R_{21}$ by Simon \& Lee.

It is also known that small amplitude Cepheids generally have more symmetrical light curves than those of large amplitude. Therefore, a relation is expected between $R_{21}$ and amplitude. We show in Sect. 6 that this indeed is the case, and as a consequence, that the amplitudes of Cepheids in the upper left panel of Fig. 6 are a continuous function of the place within the scatter of $R_{21}$ at a given period for $\log P<1$ (Fig. 13 later in Sect. 6.4). $R_{21}$ is largest (highest asymmetry) at large amplitude for all period intervals from 3 to 7 days and again from 13 to greater than 20 days, explaining the scatter at a given period in the upper left of Fig. 6.

We also show (Fig. 14 in Sect. 6.4) that $R_{21}$ varies systematically with the $(B-V)^{0}$ color when the data are binned in intervals of absolute magnitude. These correlations prove that $R_{21}$ varies systematically with position in the instability strip, just as is do amplitude and color at fixed absolute magnitude. These correlations are nearly identical in character with the same systematic correlations for RR Lyrae variables in globular clusters where the instability strip is cut at nearly constant $V$ absolute magnitude by the horizontal branch.

The interpretation of the $\Phi_{21}$ phase parameter is not as clear, but the variations shown in Fig. 6 (right panel), and from conversations with Norman Simon, suggest that $\Phi_{21}$ measures in some approximate way the progression of the "Hertzsprung" (1926) bump, occurring before maximum light near 7 day periods to after maximum for periods near 13 days. The discontinuity in $\Phi_{21}$ near 10 days is again pronounced.

A behavior very similar to LMC is known for Galactic Cepheids (Simon \& Lee 1981; Simon \& Moffett 1985; Fernie \& Ehlers 1999; Zakrzewski et al. 2000), where the variation in $\Phi_{21}$ is even somewhat larger and where again a few

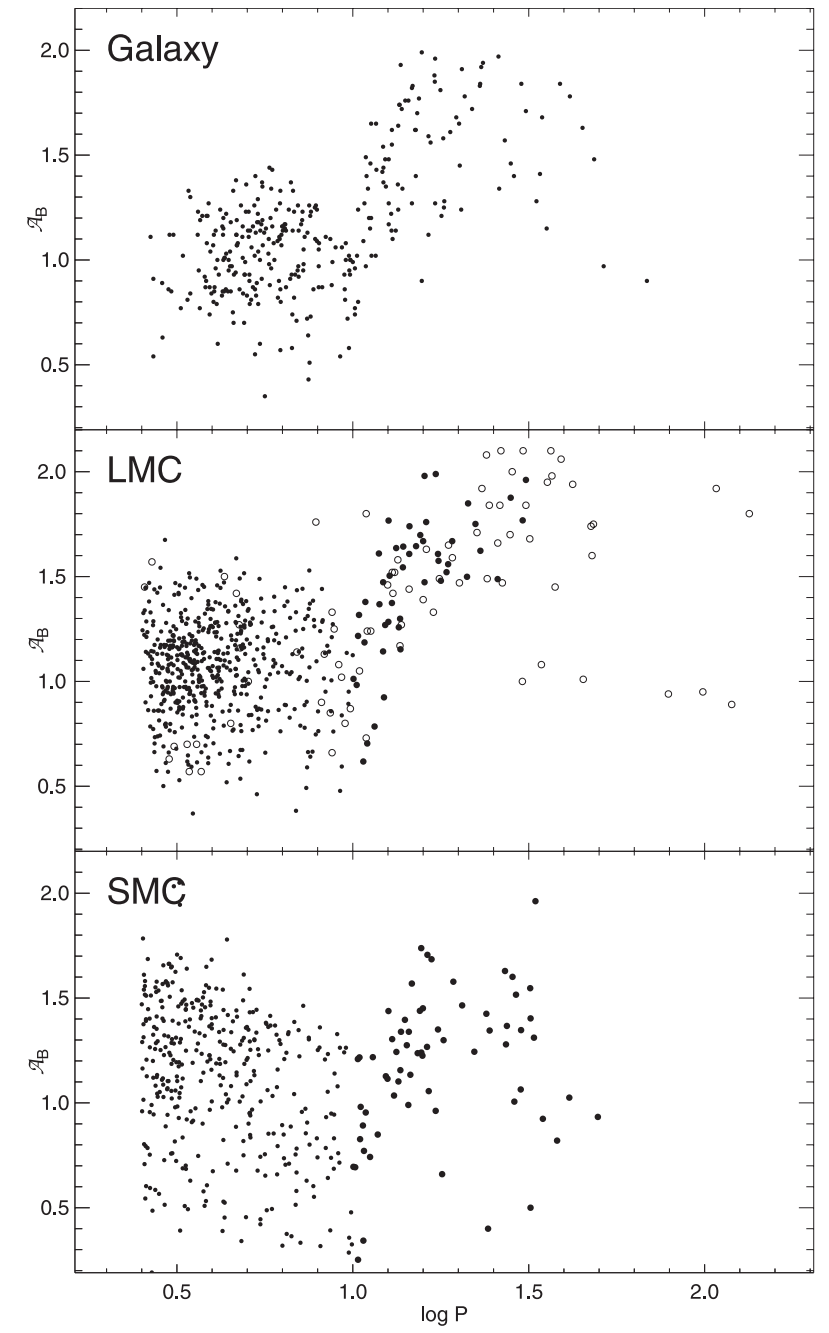

Fig. 7. The distribution of the $B$ amplitude $\mathcal{A}_{B}$ over period for the Galaxy (upper panel), LMC (middle panel), and SMC (lower panel). Symbols for LMC as in Fig. 1a.

Cepheids with $\Phi_{21} \lesssim 0.3$ are known. Simon \& Moffett (1985) note also that in some phase-phase diagrams (particularly $\Phi_{31}$ vs. $\Phi_{21}$ and $\Phi_{41}$ vs. $\left.\Phi_{31}\right)$ a clear separation of short- and longperiod Cepheids in the Galaxy exists, and they favor the idea "that the Cepheids with periods above ten days are somehow "different" from those with periods below ten days".

(2) An amplitude $-\log P$ plot of LMC Cepheids is shown in Fig. 7. The $B$ amplitudes $\mathcal{A}_{B}$ are derived from the U99 and Table 1 samples. For comparison, corresponding plots are also shown for the Galaxy (Berdnikov et al. 2000) and SMC with $\mathcal{A}_{B}$ derived from data in Udalski et al. (1999c). In all three galaxies the maximum values of the amplitudes for $\log P<0.9$ scatter widely to reach a rather pronounced minimum just shortwards of $\log P=1$ and then to increase sharply beyond this point. The characteristic dip of the maximum amplitudes around $\log P \gtrsim 1$ is common to all three galaxies (see also Schaltenbrand \& Tammann 1970; Berdnikov \& Ivanov 1986), except that the dip shortwards of $\log P=1$ is less pronounced in LMC. LMC Cepheids in general have somewhat larger amplitudes than in the Galaxy. - The amplitudes $\mathcal{A}_{1}$ to $\mathcal{A}_{4}$ of the 


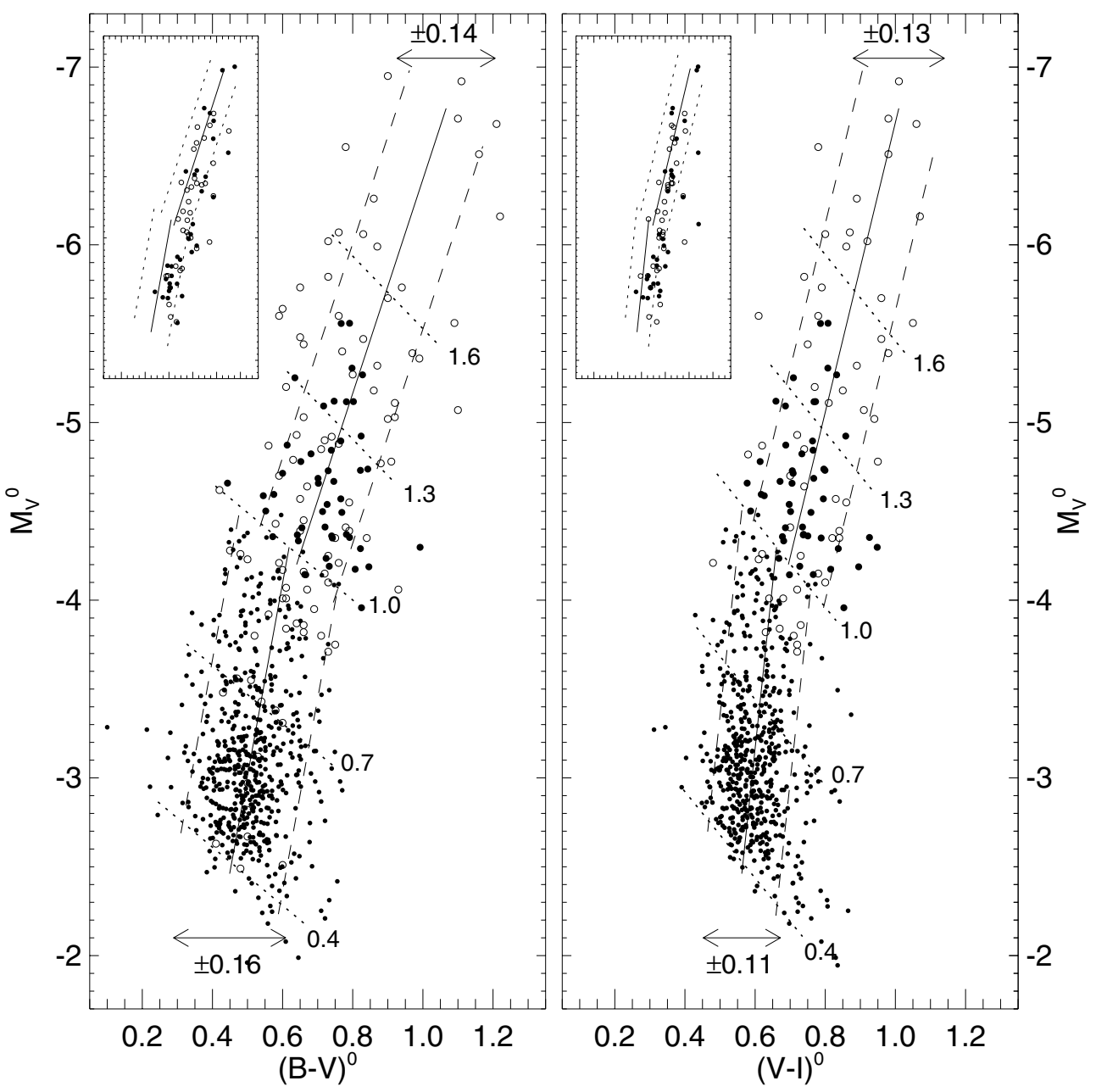

Fig. 8. The CMDs with $M_{V}^{0}$ versus $(B-V)^{0}$ and $M_{V}^{0}$ versus $(V-I)^{0}$. The ridge lines of the instability strip, resulting from Eqs. ((2), (3), (5), (6), (12) and (13)) are shown for $\log P \gtrless 1.0$. The $679\left(647\right.$ for $\left.(V-I)^{0}\right)$ dereddened sample Cepheids are plotted. Symbols as in Fig. 1a. Approximate blue and red edges of the instability strip are fitted to the data (see text). Also shown are five constant-period lines from Sect. 6.2. The inserts repeat the adopted instability strip of LMC and show in addition the 53 Galactic Cepheids with known absolute magnitude $M_{V}$ taken from Paper I (Fig. 15 there).

individual Fourier components in $V$ and $I$, plotted against $\log P$, show patterns quite similar to Fig. 7 (Ngeow et al. 2003).

(3) We show in the next section that the slope of the ridge line of the instability strip of the color-magnitude diagram (Fig. 8) has a break at $\log P=1$.

(4) Additional, unquestionable changes of the properties of short- and long-period Cepheids in LMC are shown in the next Section, where the slope of the constant-period lines suddenly steepens at $\log P=1$, where the trend of the amplitudes to decrease with increasing color $(B-V)^{0}$ becomes chaotic, and where the behavior of the Fourier coefficients $R_{21}$ and $\Phi_{21}$ changes across the instability strip. Kanbur \& Ngeow (2004) have also made the interesting observation that the Galactic $\mathrm{P}-\mathrm{C}$ relation, which is linear at mean light, flattens significantly beyond $\log P=1$ at maximum light and marginally so at minimum light. Thus lineary at mean light would be coincidental.

(5) Also the color-amplitude relations, i.e. $(V-I)^{0}$ vs. $\mathcal{A}_{V}$ at maximum, mean, and minimum light are steeper for longperiod than for short-period LMC Cepheids; the same effect is also observed in SMC and - less pronounced - in the Galaxy (Kanbur \& Ngeow 2004).

The conclusion is that several parameters and various correlations between these parameters to be set out in the next sections change their behavior near a break point at $\log P=1$. This holds for the Cepheids in LMC and SMC as well as in the Galaxy. The existence of the break therefore does not seem to be a question of metallicity, but rather to indicate that shortand long-period Cepheids of given metallicity form a nonhomologous sequence.

Another phenomenon seemingly supporting the change of character of Cepheids near $P \approx 10$ days does not withstand the analysis of modern data. It had been suggested that the number of Cepheids in the Galaxy (and in M31) is exceptionally low at the break period of $\log P=1$ (Becker et al. 1977), which was supported by Buchler et al. (1997). However, the distribution of Berdnikov's et al. (2000) large sample of Galactic Cepheids shows only a mild minimum at somewhat lower periods $(\log P=0.925)$. In the case of LMC and SMC Becker et al. (1977), using very large Cepheid samples, did not see 
any significant dip, and this is confirmed by the well searched OGLE fields in LMC and SMC (Antonello et al. 2002). The latter authors make also plausible that the overtone pulsators should be included in the frequency histograms plotting their presumed original fundamental periods.

An additional distinction of the break period is that the fundamental mode and the second-overtone mode are in resonance, i.e. $P_{0}=2 P_{2}$, for Cepheids with a period of ten days, as pulsation calculations show (Simon \& Lee 1981; Moskalik et al. 1992). Ten-day Cepheids with the metallicity of LMC have during the second crossing of the strip masses between roughly $6.5 M_{\odot}$ (red edge) and $7.8 M_{\odot}$ (blue edge) (Alibert et al. 1999). This mass range embraces - coincidentally(?) - the mass above which stars develop no degenerate core avoiding thus a helium flash, but leading eventually to a supernova explosion (Woosley \& Weaver 1986).

\section{Properties of the instability strip}

\subsection{The instability strip in the $M_{V}$ vs. $(B-V)^{0}$ and $M_{V}$ vs. $(V-I)^{0}$ plane (the $C M D$ )}

The absolute magnitudes $M_{V}^{0}$, based on an adopted value of $(m-M)_{\mathrm{LMC}}^{0}=18.54$, of 679 Cepheids are plotted against their dereddened colors $(B-V)^{0}$ and $(V-I)^{0}$ in Fig. 8. The data outline the instability strip with considerable clarity. In particular it is obvious that the ridge line of the strip is non-linear. However, the points are well represented by two linear fits whose slopes hold for the cases $\log P \gtrless 1$.

The ridge lines shown in Fig. 8 are determined by combining the P-C relations (Eqs. (2), (3) and (5), (6)) with the P-L relations in $V$ (Eqs. (12) and (13)). The resulting ridge line equations are in $(B-V)^{0}$ for

$$
\begin{array}{ll}
\log P<1: & M_{V}^{0}=-(10.83 \pm 0.98)(B-V)^{0}+(2.41 \pm 0.38) \\
\log P>1: & M_{V}^{0}=-(6.02 \pm 0.60)(B-V)^{0}-(0.35 \pm 0.36)
\end{array}
$$

and in $(V-I)^{0}$ for

$\log P<1: \quad M_{V}^{0}=-(18.56 \pm 2.58)(V-I)^{0}+(7.97 \pm 1.32)$

$\log P>1: \quad M_{V}^{0}=-(8.15 \pm 0.88)(V-I)^{0}-(1.46 \pm 0.50)$.

The difference of slope between short- and long-period Cepheids has a $4 \sigma$ significance in $(B-V)^{0}$ and $(V-I)^{0}$. - It may be noted that a determination of the ridge line through a direct regression of $M_{V}$ on color $(B-V)^{0}$ or $(V-I)^{0}$ would be inappropriate because the slopes of the constant-period lines define a highly biased sample in the CMD. The resulting slope would be much too steep particularly for the short-period Cepheids.

The blue and red boundaries of the instability strip in $(B-V)^{0}$ are drawn by eye such that the strip contains $\sim 90 \%$ of the points. The corresponding strip half-widths are 0.14 below and 0.16 above $\log P=1$.

Once the strip width in $(B-V)^{0}$ is fixed, the width in $(V-I)^{0}$ follows by necessity. This is because the absolute magnitude $M_{V}$ changes by $\Delta M_{V}$ as one goes from the blue edge to the red edge along a constant-period line. The magnitude difference $\Delta M_{V}$ must be the same in the $(B-V)^{0}$ and $(V-I)^{0}$ strips. Therefore the strip width is inversely proportional to the slopes of the constant period lines, i.e. 1.69:2.43 for shortand 2.13:2.23 for long-period Cepheids (for the slope $\beta$ of the constant-period lines see Table 4). The resulting strip halfwidths in $(V-I)^{0}$ are $\pm 0^{\mathrm{m}} \cdot 11$ and $\pm 0^{\mathrm{m}} \cdot 13$, respectively. They are shown in Fig. 8. The deduced blue and red boundaries in $(V-I)^{0}$ comprise the observed points quite well, except an unexplained number of faint Cepheids $\left(M_{V}>-3.0\right)$ which are quite red $(V-I>0.7)$. - The somewhat narrower strip width in $(V-I)^{0}$ than in $(B-V)^{0}$ is consistent with the marginally smaller scatter of the $\log P-(V-I)^{0}$ relation (Fig. 1b) than in the $\log P-(B-V)^{0}$ relation (Fig. 1a).

An important question is whether the LMC Cepheids in Fig. 8 outline the instability strip itself, or whether they only show the distribution of the Cepheids within a possibly much wider complete instability strip. The latter possibility has often been discussed because the loops of present evolutionary tracks of low-mass Cepheids (e.g. Baraffe \& Alibert 2001) do not cross the entire instability strip, but only feed its red part. This would have far-reaching consequences; e.g. one could postulate that the true instability strip was wider than shown in Fig. 8 and perfectly linear over the entire period range, and that its apparent break at $\log P=1$ were merely a reflection of how the evolutionary tracks populate the strip (Simon \& Young 1997).

However, there are three arguments which suggest that the short-period Cepheids in Fig. 8 outline the true LMC instability strip at constant period. (1) If any blue Cepheids at low luminosities were missing one would expect a blue cutoff in the $\log P-(B-V)^{0}$ relation (Fig. 1a) for say $\log P<0.6$. This is not seen. (2) It will be shown in Sect. 6.3 that the amplitudes $\mathcal{A}_{B}$ at any given period are largest close to the blue boundary of the strip, and as seen in Fig. 7 the values of $\mathcal{A}_{B}$ of the Cepheids with the shortest periods are larger, if anything, than the amplitudes of Cepheids with intermediate periods. With other words, the amplitudes of the LMC Cepheids with $0.4<\log P<0.5$ are fully consistent with their being close to the true blue boundary of the strip (cf. Fig. 11). (3) The observed width of the strip in temperature (Fig. 20) is $\Delta \log T_{\mathrm{e}}=0.08$, which is the adopted width in most models (cf. SBT). - If it is indeed correct that LMC Cepheids with $\log P=0.4$ reveal their true distribution in the instability strip, one can draw two conclusions. (1) A Cepheid with $\log P=0.4$ at the blue strip boundary has a mass of about $4.8 M_{\odot}$ (SBT, Tables 1-5; Alibert et al. 1999, Table 7). It must cross the entire instability strip during its second excursion into the strip. (2) A Cepheid with $\log P=0.4$ at the red boundary has a mass of only about $2.9 M_{\odot}$. The evolutionary loop of such a star must at least touch the red strip boundary. These conclusions are consistent with model calculations of Cepheids of appropriate metallicity (Saio \& Gautschy 1998; Alibert et al. 1999).

The strip width for very luminous Cepheids is poorly determined; only 22 Cepheids with $M_{V}<-5.5$ are available with known $(B-V)^{0}$, even less with $(V-I)^{0}$. Ten of these Cepheids fall outside the drawn boundaries of the $(B-V)^{0}$ strip. Moreover the ratio of Cepheids lying bluewards and redwards of the ridge line of the strip is $2: 1$ in $(B-V)^{0}$ and $3: 1$ in $(V-I)^{0}$. This can be taken as a confirmation of the model prediction (Alibert et al. 1999, Fig. 4) that the evolution of luminous 
Table 3. Two LMC Cepheids near the blue boundary of the instability strip yet with small amplitudes.

\begin{tabular}{ccccc}
\hline \hline HV & $\log P$ & $(B-V)^{0}$ & $(V-I)^{0}$ & $\mathcal{A}_{B}$ \\
\hline 170203 (SC3) & 0.838 & 0.34 & 0.54 & 0.38 \\
393051 (SC3) & 0.727 & 0.37 & 0.62 & 0.46 \\
\hline
\end{tabular}

Cepheids is much faster on the red side of the strip than on its blue side.

A comparison of the LMC and Galactic (see Sect. 4.2.1) instability strips is given by the inserts in Fig. 8. The width of the strips is the same to within the accuracy it can be determined. For Cepheids with $\log P<1$ the slope of the strip ridge line is about the same for LMC and the Galaxy, but the Galactic Cepheids are redder by $\sim 0.12$ in $(B-V)^{0}$ and $\sim 0.09$ in $(V-I)^{0}$. This color shift at $M_{V}=$ const. is not enough to separate the two strips. It lets them overlap in part. - LMC Cepheids with $\log P>1$ define a so much flatter strip ridge line that it crosses the Galactic ridge line at $M_{V} \sim-6.5$. At still higher luminosities the LMC strip is redder than in the Galaxy.

\subsection{The slope of the constant-period lines}

From the time that the fundamental pulsation theory was combined with the finite width of the instability strip (Sandage 1958) it was clear, that a blue Cepheid must be brighter than a red Cepheid of the same period. With other words the lines of constant period are sloped downward from blue to red in the CMD. The slope $\beta_{V, B-V}$ was determined from semi-theoretical considerations to be (Sandage \& Gratton 1963)

$\beta_{V, B-V} \equiv \frac{\Delta M_{V}^{0}}{\Delta(B-V)^{0}}=2.52$,

where $\Delta M_{V}^{0}$ and $\Delta(B-V)^{0}$ are the deviations of a Cepheid from the ridge lines of the P-L and $\mathrm{P}-\mathrm{C}$ relations, respectively ${ }^{4}$. Values of $\beta_{V, B-V}$ close to 2.52 were empirically confirmed by Sandage \& Tammann (1968), Sandage (1972), Martin et al. (1979) and Caldwell \& Coulson (1986), and similar values were widely used (e.g. Madore \& Freedman 1991). However, the assumption that $\beta_{V, B-V}$ is constant for all periods was shown to be inaccurate by Saio \& Gautschy (1998) who concluded from stellar evolution models and pulsation theory that $\beta$ increases with period for bolometric magnitudes.

The large present data set of LMC Cepheids allows to determine $\beta$ empirically for different period intervals. The Cepheids were binned by period as indicated in Fig. 9 and $\beta$ was determined in each bin. This was done for the CMDs with $M_{V}^{0}$ versus $(B-V)^{0}$ and $M_{V}^{0}$ versus $(V-I)^{0}$. The resulting slopes $\beta_{V, B-V}$ and $\beta_{V, V-I}$ are plotted with their error bars against $\log P$ in Fig. 10 . As can be seen in the figure the values of $\beta_{V, B-V}$ decline marginally over the interval $0.4<\log P<1$ to jump to $\beta_{V, B-V}>1.9$ for $\log P>1$. The behavior of $\beta_{V, B-V}$ can

\footnotetext{
${ }^{4}$ The deviations are defined throughout this paper as $\Delta M=M_{\mathrm{obs}}-$ $M_{\mathrm{PL}}$ and $\Delta(B-V)=(B-V)_{\mathrm{obs}}-(B-V)_{\mathrm{PC}}$.
}

Table 4. The coefficients in the P-L-C relation (Eq. (42)) for different wavelength combinations for LMC, SMC, and the Galaxy.

\begin{tabular}{rccc}
\hline \hline & \multicolumn{1}{c}{$\alpha$} & $\beta$ & $\gamma$ \\
\hline & & Galaxy & \\
$M_{V},(B-V)$ & $-3.324 \pm 0.180$ & $0.601 \pm 0.404$ & $-1.117 \pm 0.168$ \\
$M_{V},(V-I)$ & $-3.253 \pm 0.143$ & $0.666 \pm 0.410$ & $-1.266 \pm 0.241$ \\
\hline$M_{V},(B-V)$ & & LMC & \\
$\log P<1$ & $-3.417 \pm 0.045$ & $1.686 \pm 0.071$ & $-1.922 \pm 0.036$ \\
$\log P>1$ & $-3.407 \pm 0.099$ & $1.948 \pm 0.169$ & $-2.034 \pm 0.097$ \\
$M_{V},(V-I)$ & & & \\
$\log P<1$ & $-3.328 \pm 0.022$ & $2.434 \pm 0.041$ & $-2.565 \pm 0.025$ \\
$\log P>1$ & $-3.300 \pm 0.101$ & $2.517 \pm 0.233$ & $-2.660 \pm 0.128$ \\
\hline$M_{V},(B-V)$ & & $\mathrm{SMC}$ & \\
$\log P<1$ & $-3.086 \pm 0.075$ & $1.701 \pm 0.126$ & $-1.948 \pm 0.056$ \\
$\log P>1$ & $-3.457 \pm 0.141$ & $1.431 \pm 0.184$ & $-1.371 \pm 0.144$ \\
$M_{V},(V-I)$ & & & \\
$\log P<1$ & $-3.313 \pm 0.053$ & $2.813 \pm 0.094$ & $-2.760 \pm 0.053$ \\
$\log P>1$ & $-3.548 \pm 0.094$ & $2.825 \pm 0.175$ & $-2.513 \pm 0.130$ \\
\hline
\end{tabular}

be approximated with sufficient accuracy for the two period intervals by

$$
\text { for } \log P<1: \quad \beta_{V, B-V}=1.69 \pm 0.07,
$$$$
\text { and for } \log P>1: \quad \beta_{V, B-V}=1.95 \pm 0.17 \text {. }
$$

The values of $\beta_{V, V-I}$ vary only little over the entire period interval and they are well represented by a mean value of

$\beta_{V, V-I}=2.43 \pm 0.04$

The corresponding constant period lines are shown for five different values of $\log P$ in the instability strip of Fig. 8 .

The slope $\beta$ of the constant-period lines together with the strip half-widths in Fig. 8 yields the predicted intrinsic half-widths of the LMC P-L relations in Fig. 4. Evaluating the appropriate values of $\beta$ from Table 4 and relations given in Sect. 7.1 yield half-widths of the P-L relation of \pm 0.43 in $B, \pm 0.28$ in $V$, and $\pm 0 . \mathrm{m} 16$ in $I$. They are almost the same for $P \gtrless 10$ days and are significantly wider than in the Galaxy (see below Sect. 4.2.1). The corresponding boundaries for LMC are shown in Fig. 4.

\subsection{Amplitude and the position within the instability strip}

The systematic behavior - or lack thereof - of the amplitudes as a Cepheid evolves from right to left, or left to right, through the instability strip is a long-standing question (see e.g. Hofmeister 1967; Payne-Gaposchkin 1974). Sandage \& Tammann (1971) have concluded from the data available at the time for the Galaxy, LMC, SMC, and M 31 that the amplitudes of Cepheids with $\log P<0.86$ are largest near the blue boundary of the strip and decrease towards the red boundary in a similar pattern as 


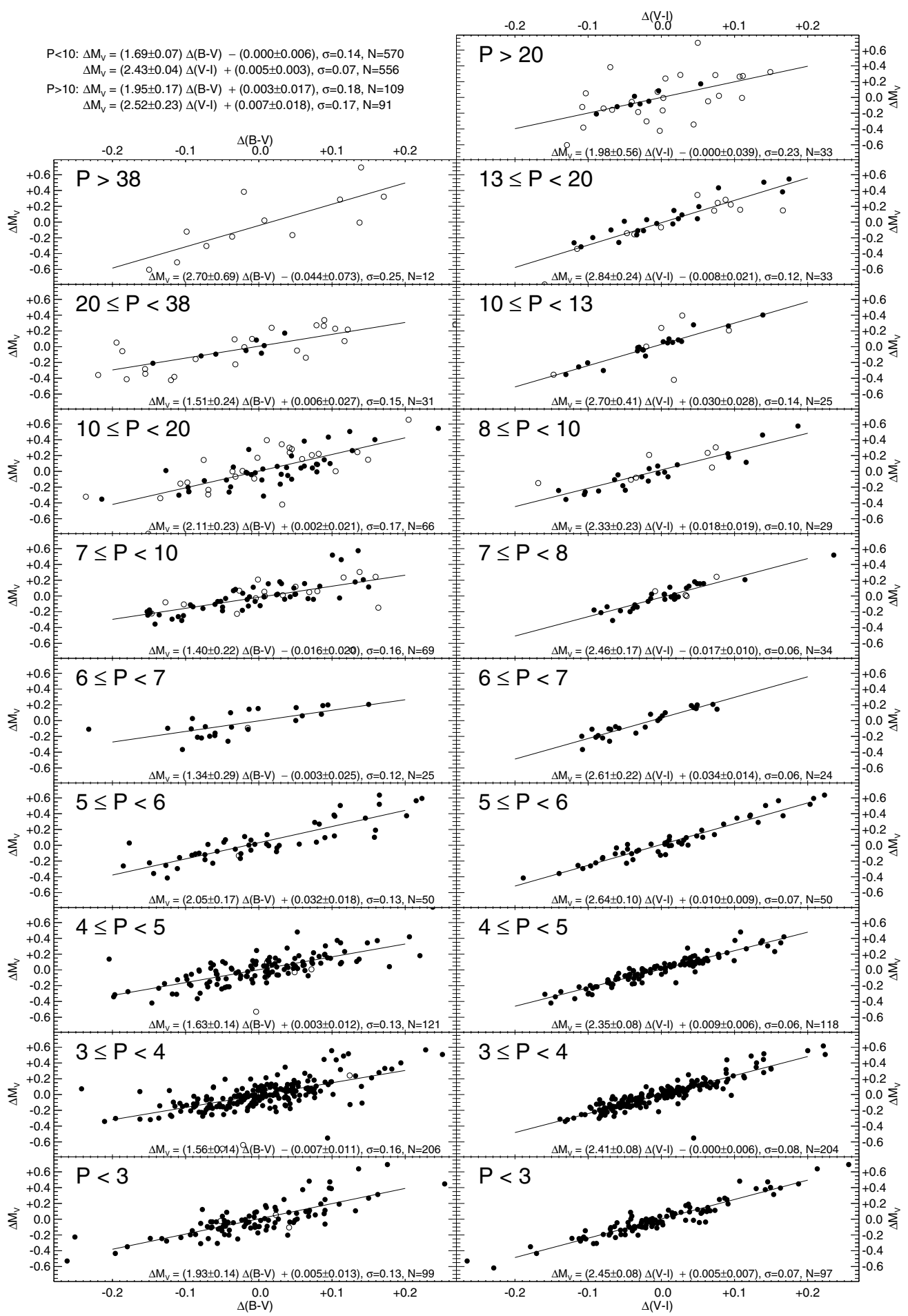

Fig. 9. The slope of the constant-period lines for different period intervals. Plotted are the magnitude residuals $\Delta M_{V}$ from the mean P-L relation (Eqs. (12) and (13)) versus the color residuals $\Delta(B-V)$ and $\Delta(V-I)$ from the respective mean P-C relation (Eqs. (2), (3), (5) and (6)). Symbols as in Fig. 1a. Positive $\Delta M_{V}$ residuals are fainter than the P-L ridge line. Positive $\Delta(B-V)$ residuals are redder than the P-C ridge line. Hence, brighter magnitudes are bluer along a constant period line.

for RR Lyrae variables. This yet unconfirmed result was less clear for longer periods in these earlier results.

The now available large sample of LMC Cepheids renders itself to a re-analysis of the amplitudes in function of the strip position.
The Cepheids are subdivided into nine period intervals in Fig. 11. Their colors $(B-V)$ are plotted against their amplitude $\mathcal{A}_{B}$. There is a definite trend of the blue Cepheids having larger amplitudes. The slope is steepest in the interval $3<P<10^{\text {d }}$; it may be somewhat flatter for $P<3^{\text {d }}$. 


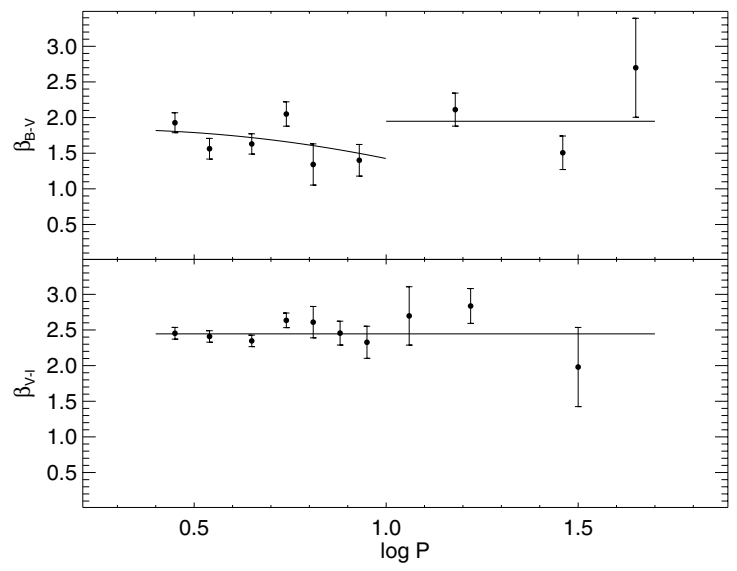

Fig. 10. The LMC slopes $\beta_{V, B-V}$ and $\beta_{V, V-I}$ of the constant-period lines in the CMD plotted against $\log P$. The mean lines are drawn by eye.

Above $P=10^{\mathrm{d}}$ the color-amplitude relation becomes marginal:

for $3<P<10^{\mathrm{d}} \quad(N=464,453)$

$\Delta(B-V)=(-0.109 \pm 0.016) \mathcal{A}_{B}+0.117 \pm 0.018, \sigma=0.08$

$\Delta(B-V)=(-0.238 \pm 0.023) \mathcal{A}_{V}+0.173 \pm 0.017, \sigma=0.08$

and for $P>10^{\mathrm{d}} \quad(N=99,79)$

$\Delta(B-V)=(-0.059 \pm 0.029) \mathcal{A}_{B}+0.087 \pm 0.044, \sigma=0.10$

$\Delta(B-V)=(-0.121 \pm 0.047) \mathcal{A}_{V}+0.108 \pm 0.045, \sigma=0.10$.

The data in Paper I give similar relations between the color residuals $\Delta(B-V)$ and amplitude $\mathcal{A}_{B}$ for the Galaxy, i.e.

for $3<P<10^{\mathrm{d}}$

$\Delta(B-V)=(-0.113 \pm 0.017) \mathcal{A}_{B}+0.115 \pm 0.018$,

and for $P>10^{\mathrm{d}}$

$\Delta(B-V)=(-0.043 \pm 0.031) \mathcal{A}_{B}+0.062 \pm 0.046$.

Two of the 563 Cepheids in Fig. 11 are near to the blue boundary as drawn in Fig. 8 and have exceptionally small amplitudes. Their parameters are given in Table 3 . They seem to belong to the rare class of blue Cepheids with small, rapidly changing amplitudes. Of course, the possibility remains that they are instead overtone pulsators.

The color-amplitude relations in Fig. 11 clearly show that, statistically, the amplitudes are largest near the blue side of the instability strip, decreasing toward the red along lines of constant period, except for the period bin of 10 to 20 days, where the relation changes sign. This was the original result of Sandage \& Tammann (1971) where they concluded that for $0.4<\log P<0.86$, the amplitude is largest near the blue edge of the strip, decreasing toward the red. But in the period interval $0.86<\log P<1.3$ the trend is reversed, returning to the original sense for $\log P>1$.3. Figure 11 confirms this early result, based now on a much larger sample than was available in 1971 .

It is easier to visualize the trends in the strip by binning the data by absolute magnitude rather then, as in Fig. 11, by period. This maps the amplitude properties of the strip horizontally in the HRD (i.e. nearly at constant absolute magnitude). This is the way the properties of the RR Lyrae instability strip

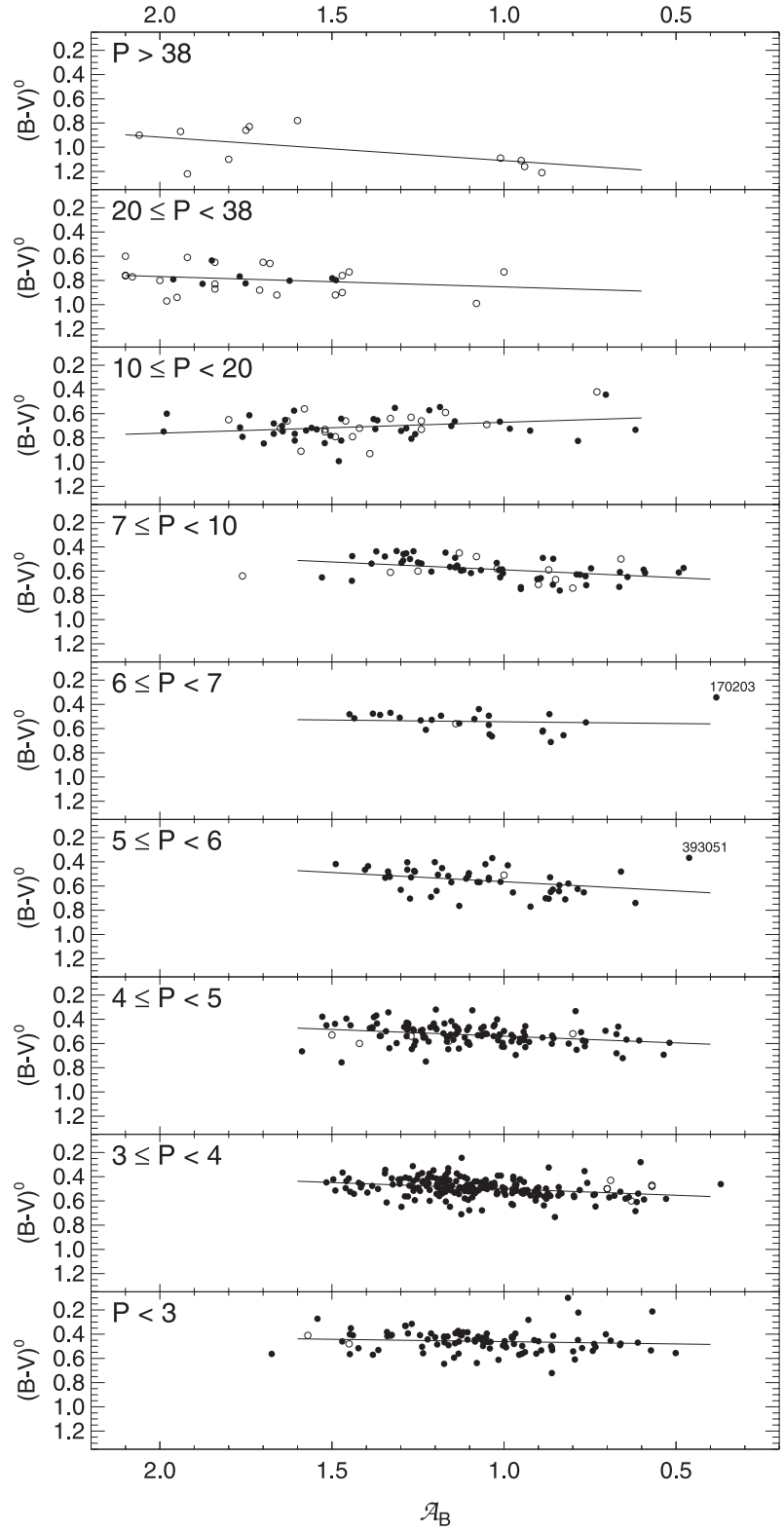

Fig. 11. The correlation of the color $(B-V)$ and the $B$ amplitudes $\mathcal{A}_{B}$ for different period intervals. The largest amplitude occur near the blue boundary of the instability strip, except in the range $10<P<20^{\mathrm{d}}$. Symbols as in Fig. 1a.

has traditionally been mapped because the horizontal branch of the clusters cuts the instability strip at nearly constant absolute $V$ magnitude.

Figure 12 shows such a binning in five intervals of absolute magnitude ranging from $M_{V}=-2.75$ to $M_{V}<-4.75$. Clearly the largest amplitudes occur at the blue side of the instability strip for all absolute magnitudes except, again, in the magnitude range where the periods are near 10 days $\left(-4.25>M_{V}>\right.$ $-4.75)$. Here the relation becomes chaotic or perhaps even reversed as in Sandage \& Tammann (1971).

Because amplitude varies systematically with color across the strip (Fig. 11) and because of the correlation between color residuals $\Delta(B-V)$ and magnitude residuals $\Delta M_{V}=$ $M_{V}^{0}-M_{V \text {,ridge }}$ (Fig. 9) there must be also a correlation between 


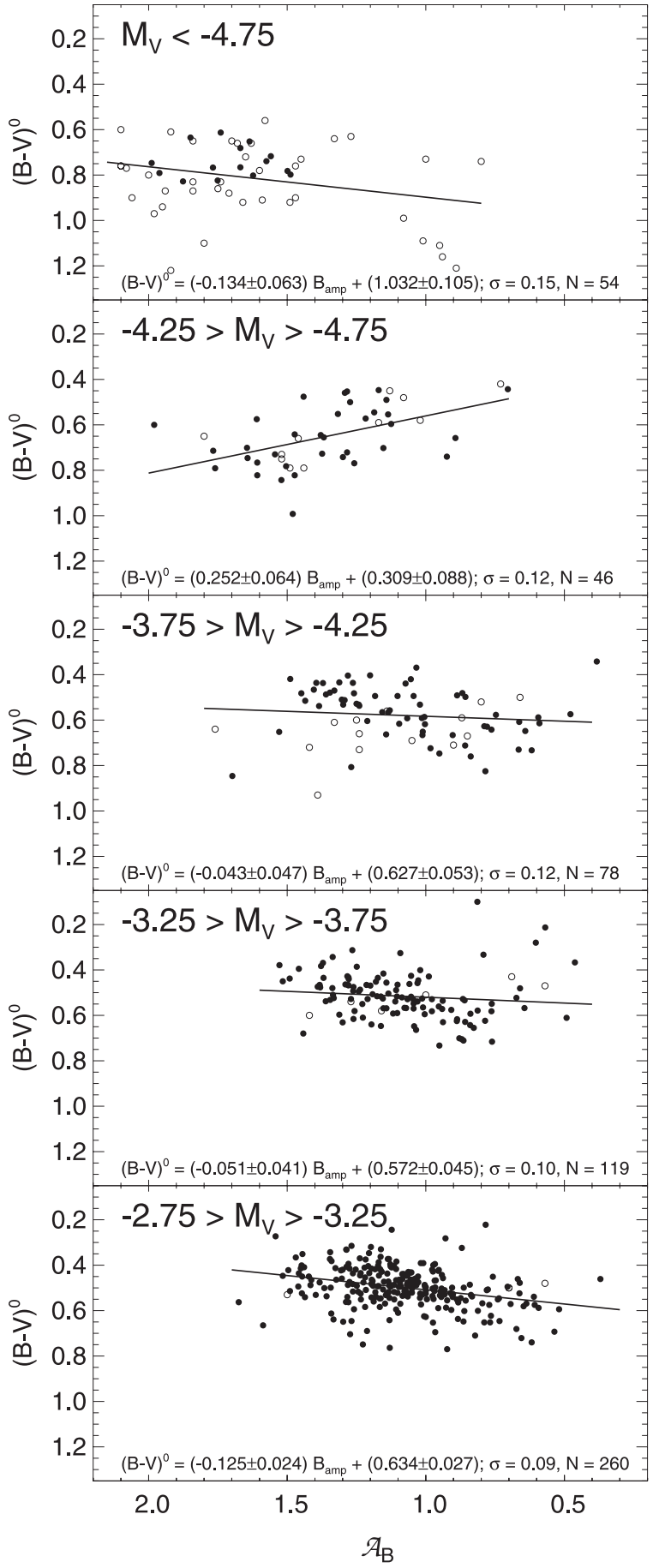

Fig. 12. The color-amplitude relation in five intervals of absolute magnitude showing a regular progression except in absolute magnitude interval of $-4.25>M_{V}>-4.75$ which is in the period interval near 10 days. Symbols as in Fig. 1a.

magnitude residuals $\Delta M$ and amplitudes. By means of a diagram analogous to Fig. 11 the relation is found to be

$$
\begin{aligned}
& \text { for } 3<P<10^{\mathrm{d}} \quad(N=464,453,452) \\
& \Delta M_{V}=(-0.223 \pm 0.040) \mathcal{A}_{B}+0.239 \pm 0.043, \sigma=0.20 \\
& \Delta M_{V}=(-0.479 \pm 0.055) \mathcal{A}_{V}+0.352 \pm 0.041, \sigma=0.18 \\
& \Delta M_{I}=(-0.066 \pm 0.024) \mathcal{A}_{B}+0.072 \pm 0.027, \sigma=0.13
\end{aligned}
$$

and for $P>10^{\mathrm{d}} \quad(N=99,79,82)$

$\Delta M_{V}=(-0.091 \pm 0.073) \mathcal{A}_{B}+0.146 \pm 0.113, \sigma=0.25$

$\Delta M_{V}=(-0.160 \pm 0.121) \mathcal{A}_{V}+0.146 \pm 0.117, \sigma=0.26$

$\Delta M_{I}=(-0.005 \pm 0.057) \mathcal{A}_{B}+0.008 \pm 0.082, \sigma=0.19$.

With these equations one can derive period-luminosityamplitude relations, but their practical use remains restricted even for the short-period interval $3<P<10^{\text {d }}$, because the scatter is hardly reduced compared to the straight P-L relations (Eqs. (12) and (13)).

Equations (36)-(41) express a warning against systematic distance errors. The importance of unbiased Cepheid samples has been pointed out by several authors (e.g. Sandage 1988; Paturel et al. 2002). Small-amplitude ( $\mathcal{A}_{B} \approx 0$ m 5 ) Cepheids turn now out to be fainter on average than their long-period counterparts $\left(\mathcal{A}_{B} \approx 2^{\mathrm{m}}\right)$ by $\sim 0^{\mathrm{m}} 2$. To the extent that small amplitude Cepheids are harder to detect, they may be underrepresented in available Cepheid samples. Yet a fair amplitude distribution here turns out to be prerequisit of an unbiased distance determination. (Because of the correlation of amplitude with color, a fair color distribution could also assure an unbiased Cepheid sample, but dust reddening and typically large color errors make this kind of test notoriously difficult.)

To estimate the amplitude bias we consider first the period interval $3<P<10^{\mathrm{d}}$ and make the rather extreme assumption that in an external galaxy like LMC only the 50 Cepheids with the largest amplitudes (out of a total of 464) were discovered. Their median amplitude is $\mathcal{A}_{B}=1.51$ to be compared with the true median value of $\mathcal{A}_{B}=1.09$. If the amplitude difference $\Delta \mathcal{A}_{B}=0.42$ is inserted in Eq. (36) we find that the detected sample is overluminous by 0.09 , corresponding to a distance overestimate of $4 \%$. - For the interval $P>10^{\mathrm{d}}$ the situation is still more favorable because the coefficient of $\mathcal{A}_{B}$ in Eq. (39) is about three times smaller and in fact insignificant. Because essentially all known Cepheids outside the Local Group have periods larger than $10^{\mathrm{d}}$, the amplitude bias affects the published Cepheid distances beyond $\sim 1 \mathrm{Mpc}$ only at the $1 \%$ level. In any case amplitude bias cannot be the cause of the large bias of Cepheid distances suggested by Teerikorpi \& Paturel (2002).

\subsection{Variations in the $R_{21}$ and $\Phi_{21}$ Fourier components across the strip at constant absolute magnitude}

The position of a Cepheid in the strip at a given absolute magnitude is, of course, measured by its unreddened color. Therefore, a systematic trend of any property (amplitude, period, asymmetry, bump position relative to the maximum phase, etc.) with color, maps that property across the strip.

$R_{21}$ is a strong function of amplitude, proved in Fig. 13 (right panel) where the data are binned in small intervals of period so as to understand the scatter in Fig. 6 (left panel) in $R_{21}$ at a given period. The correlations between $R_{21}$ and amplitude are strong. High values of $R_{21}$ (high asymmetry in the light curve) go with high amplitude. Hence, following the discussion of Fig. 6 in Sect. 5, the lower envelope to the $R_{21}$-period distribution is populated by low amplitude variables and the upper envelope by high amplitude variables in each period interval. 


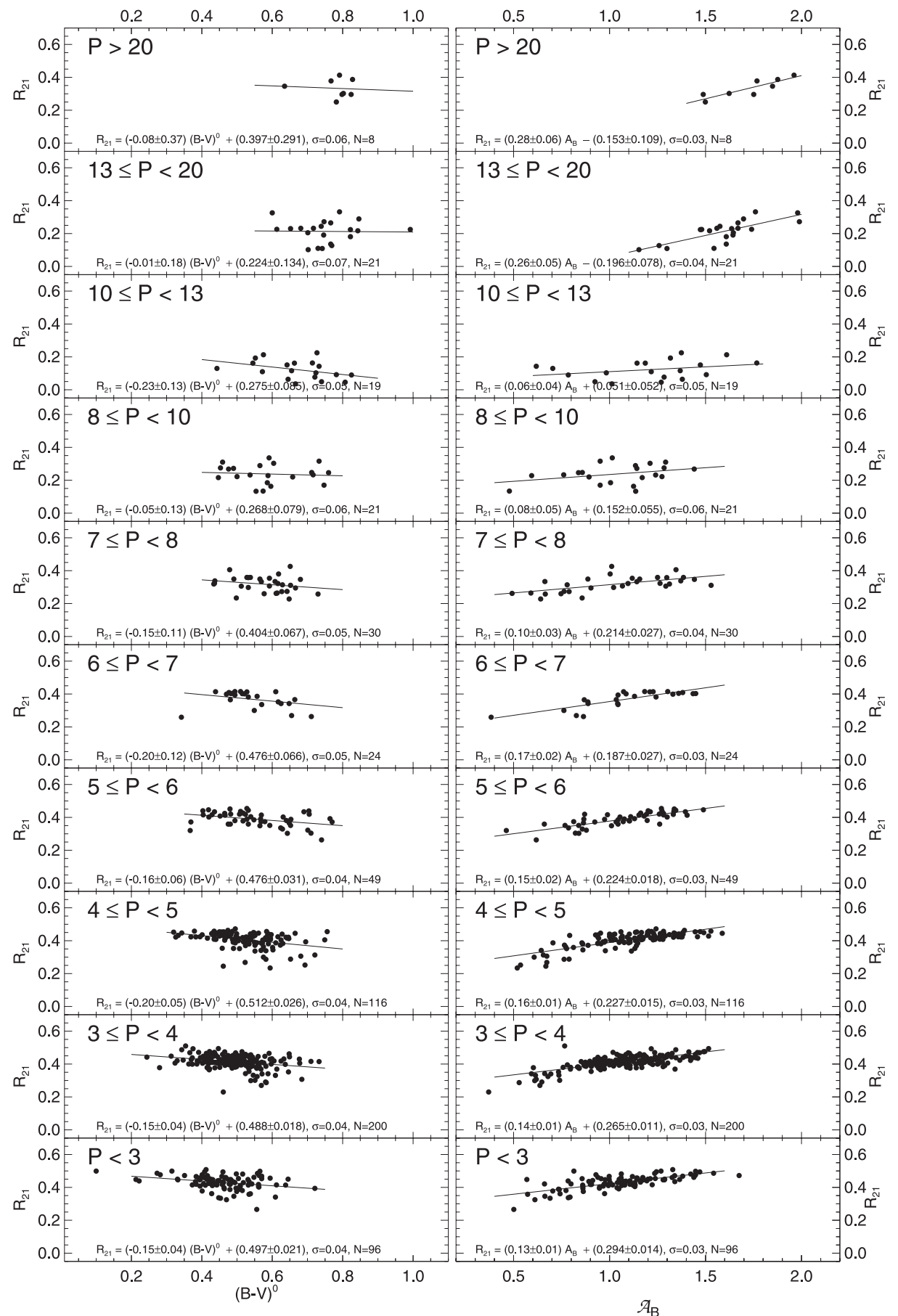

Fig. 13. Correlations of $R_{21}$ with the $(B-V)^{0}$ color (left) and with the amplitude $\mathcal{A}_{B}$ of the $B$ light curves (right) in narrow bins of period. High $R_{21}$ values occur at high amplitude and blue color, showing that $R_{21}$ varies systematically across the instability strip, with highest values near the blue edge of the strip.

Because $R_{21}$ is such a strong function of amplitude, and because amplitude varies systematically with color across the strip (NB again; high amplitude variables are bluest), there must be a correlation of $R_{21}$ with color. If so, $R_{21}$ is expected to be largest at bluest colors at a given period. The left panel of Fig. 13 shows this to be the case. And, again, by analogy with the correlations known for the RR Lyrae variables at constant absolute magnitude, the above conclusion is again evident in Fig. 14 (left column) where $R_{21}$ values are correlated with $(B-V)^{0}$ color at constant absolute magnitude. The highest $R_{21}$ values (highly peaked light curves) occur in all absolute magnitude intervals at the bluest colors. Clearly, $R_{21}$ varies systematically across the gap at all absolute magnitudes.

The meaning of the systematic variation of $\Phi_{21}$ is not as clear as that of $R_{21}$, yet the right panel of Fig. 14 shows that this phase parameter also varies systematically across the strip.

To continue the analogy with the RR Lyrae correlations of parameters at constant magnitude across the RR Lyrae strip, we 


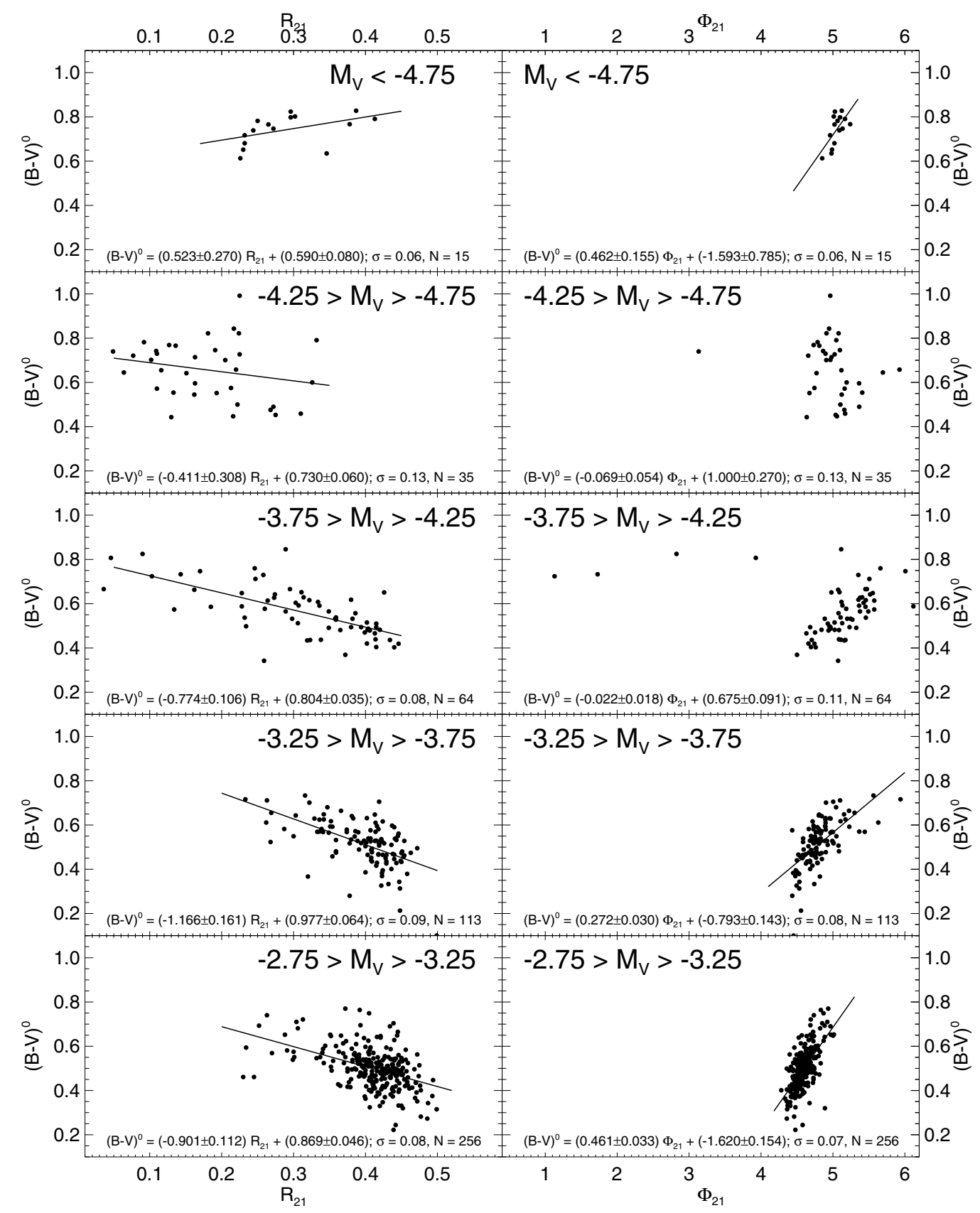

Fig. 14. Correlations of $R_{21}$ and $\Phi_{21}$ with $(B-V)^{0}$ color in narrow intervals of absolute magnitude. This maps the $R_{21}$ and $\Phi_{21}$ Fourier combinations across the strip at constant luminosity.

show in Fig. 15 the correlations between $R_{21}$ and period, again at fixed absolute magnitude. If one draws in mind's eye a horizontal line across the strip in the CMD (Fig. 8), say at absolute magnitude $M_{V}=-4$, then a family of lines of constant period (not shown) with $\log P$ between about 0.8 and 0.85 will cut across this constant absolute magnitude line at different colors within the strip boundaries. And because $R_{21}$ is strongly correlated with color (Fig. 14), necessarily, the shortest period lines (at bluer colors) will also cut the $R_{21}$ correlation with color at higher $R_{21}$ values. There must, then, be a period- $R_{21}$ relation at each fixed absolute magnitude if the lines of constant period slope in the $M_{V}$-color plane. Figure 15 shows the $R_{21}-\log P$ relation for the LMC data binned in intervals of fixed absolute magnitude. It is in the expected sense for the three faintest absolute magnitude intervals.

Because of the correlation of $\Phi_{21}$ with color in Fig. 14 (right panels) and the period-color relations at constant $M_{V}$ (Fig. 16 in the next section), $\Phi_{21}$ must necessarily be correlated with period (at constant $M_{V}$ ) for the same reasons as $R_{21}$. The right panels of Fig. 15 show this to be true, but again the interpretation in terms of the Hertzsprung "bumps" is not obvious. 


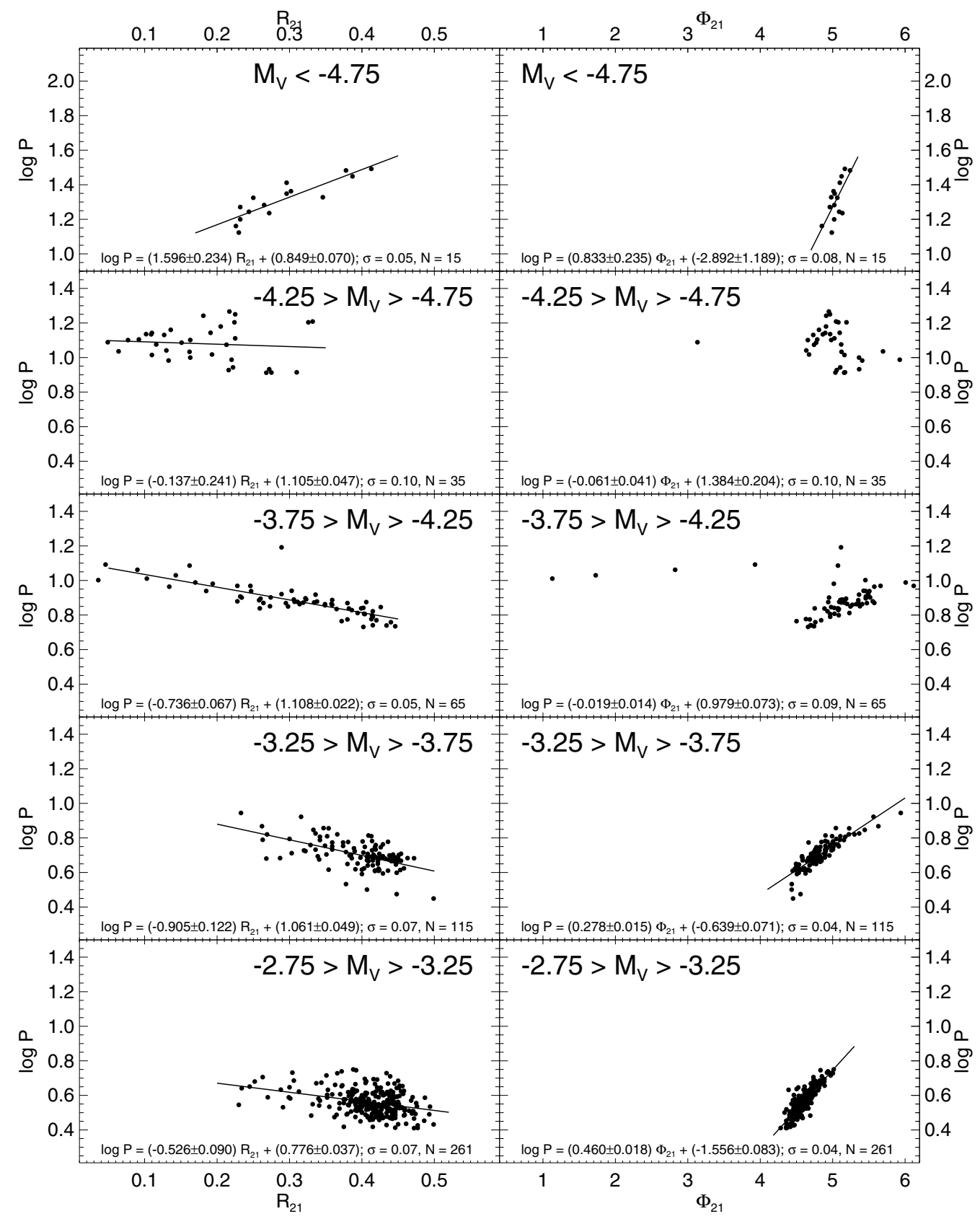

Fig. 15. The $R_{21}$ and $\Phi_{21}$ Fourier components correlated with period in intervals of absolute magnitude.

\subsection{The color-period and period-amplitude relations at constant absolute magnitude}

In analogy with the RR Lyrae correlations, and for the same reason just given (lines of constant period cutting a constant absolute magnitude line in the CMD at different color), there must be a period-color relation at constant absolute magnitude. In an obvious representation, the period-color relations in $(B-$ $V)^{0}$ and $(V-I)^{0}$ are shown in Fig. 16. As expected, and as in the RR Lyrae correlation, shorter periods occur at bluer color at fixed absolute magnitude for all absolute magnitude intervals from the faintest to the brightest. There is no break at 10 days because Fig. 9 for the lines of constant period show that the slope of these lines always has the same sense for all periods, including the intervals that embrace 10 days. If the slope had anywhere been zero or negative, then the correlations in Fig. 16 would either be flat or would slope in the opposite direction than shown.

In a similar way, again as in the RR Lyrae correlations, there are correlations between period and amplitude for fixed intervals of absolute magnitude, shown in Fig. 17. The reverse slope for the absolute magnitude interval of $-4.25>M_{V}>$ -4.75 and the reversal again to the original is a consequence of the reverse color-amplitude relations in the relevant period ranges (i.e. $\log P<0.86,0.86<\log P<1.3$, and $\log P>1.3$ ) discussed earlier (Fig. 12) and by Sandage \& Tammann (1971). 


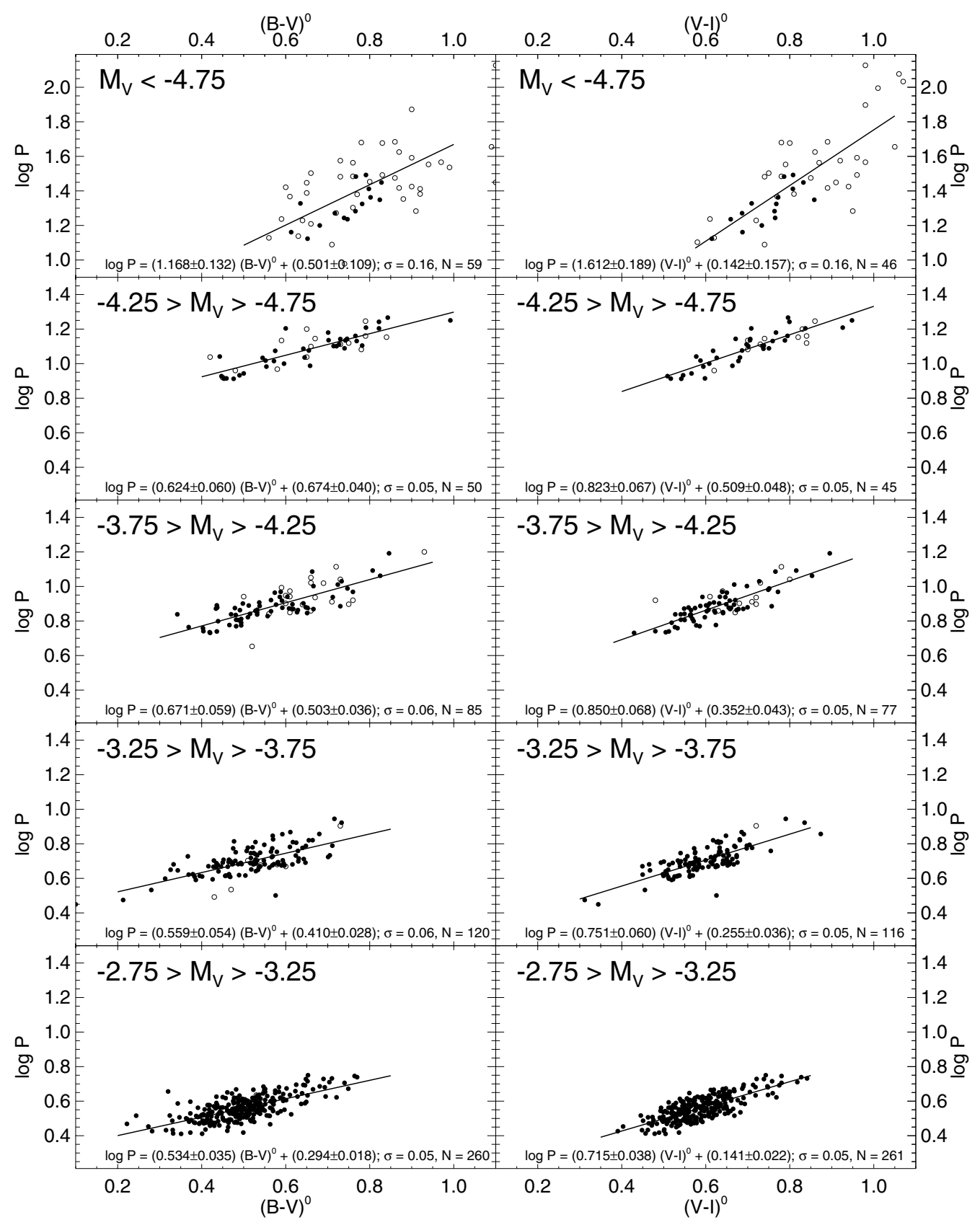

Fig. 16. The period-color correlations at fixed absolute luminosity intervals. The correlations are similar and in the same sense as for RR Lyrae stars (because the lines of constant period in the CMD have similar slopes here and for the RR Lyrae variables). Symbols as in Fig. 1a.

\section{The Period-Luminosity-Color relation of LMC}

\subsection{The $P-L-C$ relation}

The intrinsic scatter of the $\mathrm{P}-\mathrm{L}$ relation is due to the finite width of the instability strip and the sloping of the constant-period lines. The scatter can be reduced by introducing a P-L-C relation of the form

$M_{\lambda}^{\mathrm{R}}=a_{\lambda} \log P-\beta_{\lambda}[(B-V)$ or $(V-I)]+\gamma_{\lambda}$,

where $\beta$ is the slope of the constant-period lines as discussed in Sect. 6.2.

Equation (42) translates the magnitude to the value an unreddened Cepheid would have if it were lying on the ridge line of the P-C relation and hence - except for observational errors - also on the ridge line of the P-L relation. The application of the P-L-C relation has been tried many times with values of $\beta_{V, B-V} \sim 2.5$, but the results were generally unsatisfactory. The reason is now clear, because the value was too high and it is strongly variable from galaxy to galaxy as will be shown in the following.

The coefficients $\alpha, \beta$, and $\gamma$ for $M_{V}$ can be determined by combining the P-L relation (Eqs. (12) and (13)) with the appropriate P-C relations (Eqs. (2), (3), (5), and (6)) or by direct regression over the available Cepheids. Either way yields the same results with only minute differences. The results for LMC are shown in Table 4. 


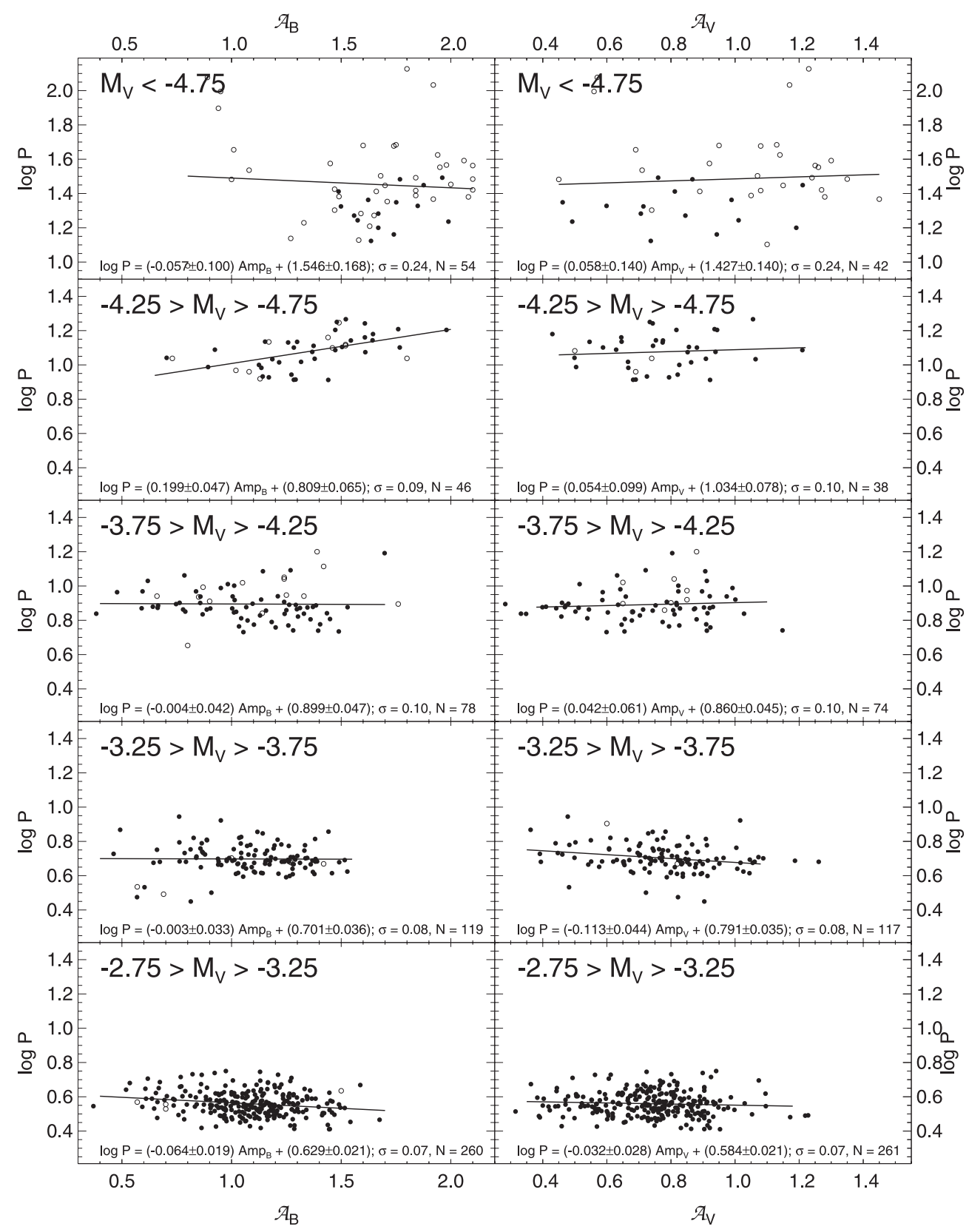

Fig. 17. The period-amplitude correlations at fixed absolute magnitude intervals as similarly known for RR Lyrae stars in globular clusters. Symbols as in Fig. 1a.

The values $\beta_{V, B-V}$ and $\beta_{V, V-I}$ transform $V$ magnitudes onto the ridge line of the P-L relation in $V$. It follows trivially that the coefficients to bring the $B$ and $I$ magnitudes onto the respective ridge lines are

$\beta_{B, B-V} \equiv \frac{\Delta M_{B}}{\Delta(B-V)}=\beta_{V, B-V}+1$

and

$\beta_{I, V-I} \equiv \frac{\Delta M_{I}}{\Delta(V-I)}=\beta_{V, V-I}-1$.

With these equations it is easy to convert the P-L-C relation in $M_{V}$ and $(B-V)$ into those in $M_{B}$ and $(B-V)$ or $M_{I}$ and $(V-I)$.

While we have for LMC $\alpha_{V, V-I}=\alpha_{I, V-I}=-3.328$ and -3.300 for short- and long-period Cepheids, respectively,
Udalski's et al. (1999a) overall value of $\alpha_{I, V-I}=-3.246 \pm$ 0.015 is slightly flatter; this is explained by the additional longperiod Cepheids used here from Table 1 . Their value $\beta_{I, V-I}=$ $1.409 \pm 0.026$, implying $\beta_{V, V-I}=2.409 \pm 0.026$, is somewhat smaller than the two corresponding values given in Table 4 for short- and long-period Cepheids.

The coefficients in Table 4 are directly determined by linear regressions in two variables. An alternative way is to combine the P-L relations as given above (Sects. 4.1 and 4.2.1; the values of SMC still being unpublished) with the appropiate P-C relations (Sect. 3 and Paper I, Eqs. (3) and (5)). This leads to the same coefficients as in Table 4 with only minute differences. In addition the coefficients $\beta$ of LMC are nearly identical - as required - with the slope of the constant period lines 


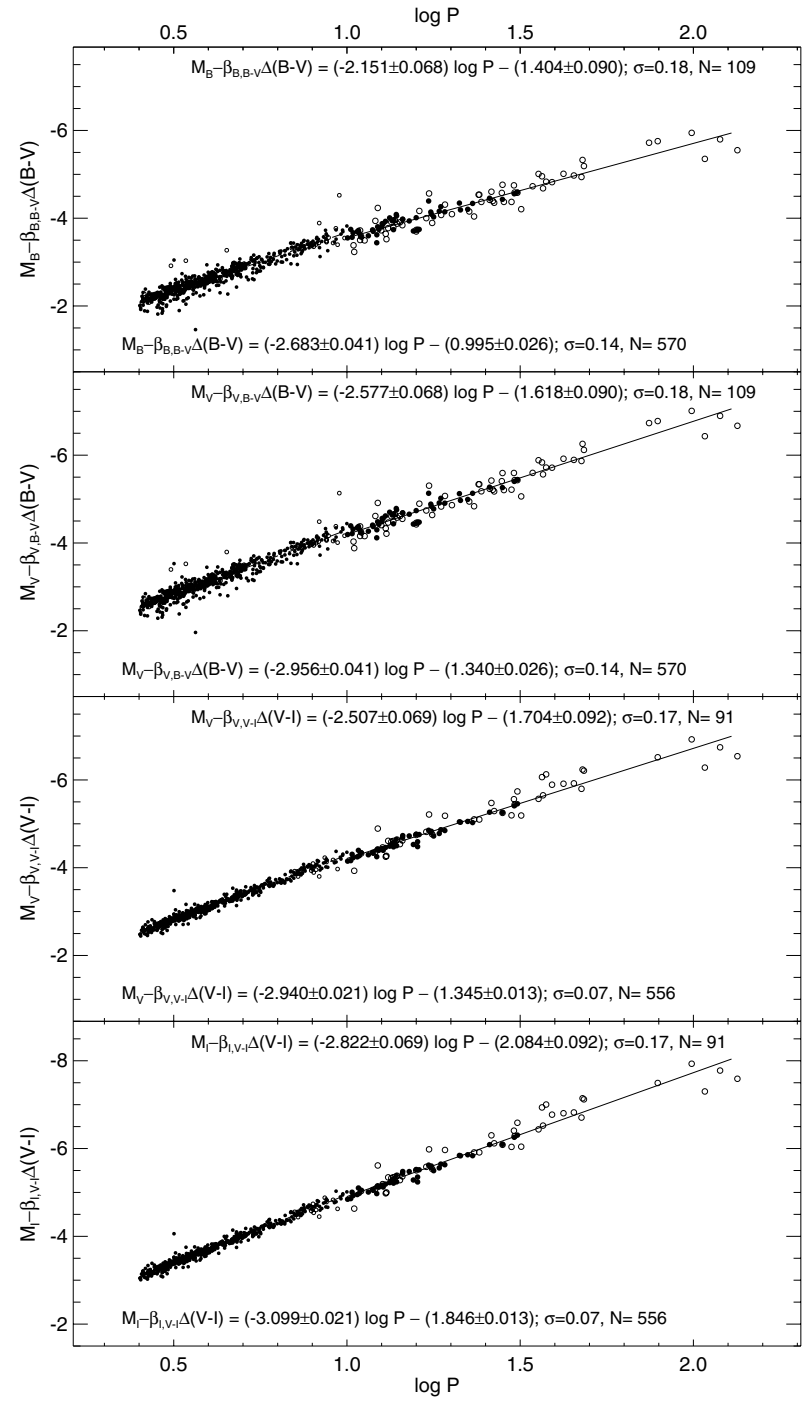

Fig. 18. The P-L relations of LMC after correcting $M_{\lambda}$ (obs) for the color term $\beta_{\lambda} \Delta(B-V)$ and $\beta_{\lambda} \Delta(V-I)$, respectively; for definitions see Sect. 6.2. The small scatter may be noted. The least-squares P-L relations for $\log P \gtrless 1.0$ are given in the lower and upper part, respectively, of each panel.

(Figs. 9 and 10, Eqs. (27)-(29)). This strengthens the confidence in the P-L-C relations of Table 4 for LMC; the same holds also for SMC. In case of the Galaxy the coefficients have large errors because they rest on only 69 calibrators, which in addition carry the errors of their individual BBW or cluster distances.

The ridge line-corrected LMC magnitudes $M_{B, V, I}^{\mathrm{R}}$ from Eq. (42) and Table 4 are plotted versus $\log P$ in Fig. 18. The $M_{V}^{\mathrm{R}}$ are plotted twice, once based on $(B-V)^{0}$ and a second time on $(V-I)^{0}$. It turns out that using the $(V-I)^{0}$ colors as second parameter leads to about half the scatter compared to using the $(B-V)^{0}$ colors. This can be understood because the simple P-L relation has considerably less scatter in $M_{I}$ than in $M_{B, V}$ (see Fig. 4), and also the scatter of the P-C relation is somewhat larger in $(B-V)^{0}$ than in $(V-I)^{0}$ (cf. Figs. 1a and 1b). Averaging the different $M^{\mathrm{R}}$ values does not reduce the scatter indicating that the errors are correlated.
The equation for the ridge line of the $\log P-M_{B, V I}^{\mathrm{R}}$ relations (shown in Fig. 18) are closely the same - as must be required - as the P-L relations in Eqs. (10)-(15). The only significant difference is that the scatter in $M^{\mathrm{R}}$ is reduced by a factor of $\sim 2$. This is of course an important practical advantage for the determination of Cepheid distances, provided the appropriate P-L-C relation is known.

Shown in Table 4 are also the coefficients of the Galactic P-L-C relation. The coefficients have here larger errors because only 69 Galactic Cepheids are available with absolute magnitudes either from cluster membership or from purely physical BBW distances (see Sect. 4.2.1). The P-L-C relation of SMC, based on data by Udalski et al. (1999c) has somewhat provisional character; a more thorough discussion is deferred to Paper III.

It can be seen in Table 4 that the coefficient $\alpha$ of the period term of the P-L-C relation varies slightly, but significantly between the Galaxy, LMC, and SMC. In contrast, the variation of the coefficient $\beta$ of the color term is very important; it is always (much) larger in LMC than in the Galaxy and is generally larger in LMC than in SMC except $\beta_{V, B-V}$ which is smaller in SMC than in LMC.

\subsection{An application of the $P-L-C$ relation}

The present LMC Cepheids being distributed along the bar of the galaxy offer the possibility to determine the space orientation of the bar. The P-L-C relation with its small scatter is ideally suited for this purpose. The moduli $\left(m_{V}-M_{V}\right)$ are calculated twice from the only two independent P-L-C relations (see Table 4)

$\log P<1.0: \quad M_{V}=-3.417 \log P+1.686(B-V)-1.922(45)$ $\log P>1.0: \quad M_{V}=-3.407 \log P+1.948(B-V)-2.034(46)$

and

$\log P<1.0: \quad M_{V}=-3.328 \log P+2.434(V-I)-2.565(47)$ $\log P>1.0: \quad M_{V}=-3.300 \log P+2.517(V-I)-2.660 .(48)$

(Note that the P-L-C relations involving $M_{B},(B-V)$ and $M_{I},(V-I)$ can be derived from the above equations and do not contain any additional information.) The resulting distances are plotted against Right Ascension in Fig. 19. As can be seen, the bar is amazingly well aligned with the plane of the sky; the eastern end of the bar is only $0.03(1.5 \%)$ closer than its western counterpart (cf. Welch et al. 1987; Caldwell \& Laney 1991).

A word of warning is in place: the P-L-C relation can only be used if it can be proven that the Cepheids under consideration follow the same P-L and P-C relations. Otherwise any intrinsic color difference is multiplied by the constant-period slope $\beta$ and erroneously forced upon the magnitudes with detrimental effects on any derived distances. It would be particularly objectionable to apply for instance the LMC P-L-C relation to Galactic Cepheids which have different $\mathrm{P}-\mathrm{L}_{B V I}$ relations and (necessarily) also different $\mathrm{P}-\mathrm{C}_{B-V, B-I}$ relations. 


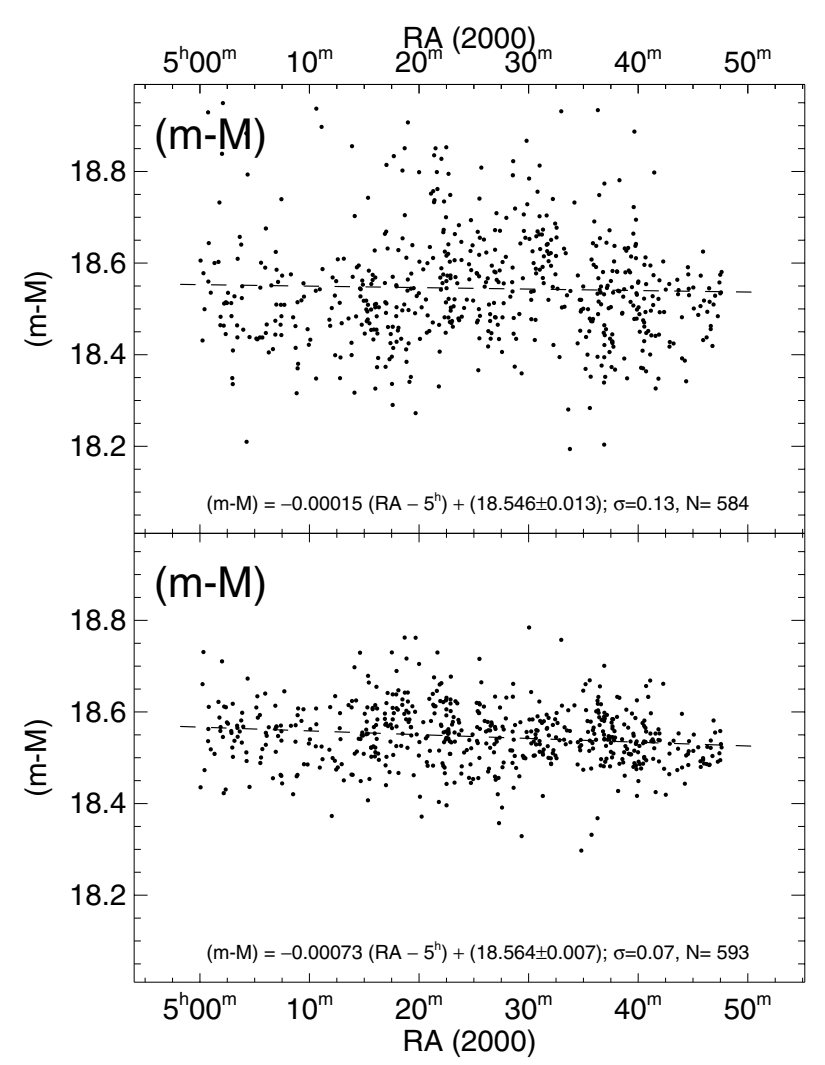

Fig. 19. The distance moduli $\left(m_{V}-M_{V}\right)$ of the individual Cepheids along the LMC bar are plotted in function of Right Ascension. Upper panel: $M_{V}$ calculated from Eqs. (45) and (46), respectively. Lower panel: $M_{V}$ calculated from Eqs. (47) and (48). The data involving $(V-I)$ show considerably less scatter than those involving $(B-V)$.

\section{The instability strip in the luminosity $-T_{\mathrm{e}}$ plane}

The instability strip in the plane of the CMD in Fig. 8 is converted into the $\log L-\log T_{\mathrm{e}}$ plane by means of the atmospheric models of Cepheids by SBT. The colors $(B-V)^{0}$ and $(V-I)^{0}$ of the ridge line and the boundary lines of the strip as well as of the individual Cepheids yield by interpolation in their Table 6 for $[\mathrm{Fe} / \mathrm{H}]=-0.3$ adopted for LMC, a turbulence of $1.7 \mathrm{~km} \mathrm{~s}^{-1}$, and variable $\log g$ two temperatures which are averaged. The dependence of $\log g$ on $\log P$ was determined from their Table 3 to be

$\log g=-1.09 \log P+2.64$,

which holds well for the ridge line as well as the blue and red boundary, and which is insensitive to metallicity (cf. their Table 2). For the luminosity $L$ (in solar units) it is assumed that $M_{\text {bol }} \approx M_{V}$ and $M_{\text {bol } \odot}=4$. 75 . The resulting diagram is shown in Fig. 20. The mean strip position as defined by Galactic Cepheids in Paper I is also shown.

It is obvious in Fig. 20 that the blueness of LMC Cepheids is due, in addition to the technical effect of blanketing, to a real temperature difference. LMC Cepheids with a luminosity of $\log L=3.5$ are hotter by about $350 \mathrm{~K}$ than their Galactic counterparts, decreasing to $\sim 80 \mathrm{~K}$ at $\log L=4.0$.

Although the temperature shift in the HR diagram of Fig. 20 is small at $\Delta \log T_{\mathrm{e}}=0.02$ compared with the total width of the

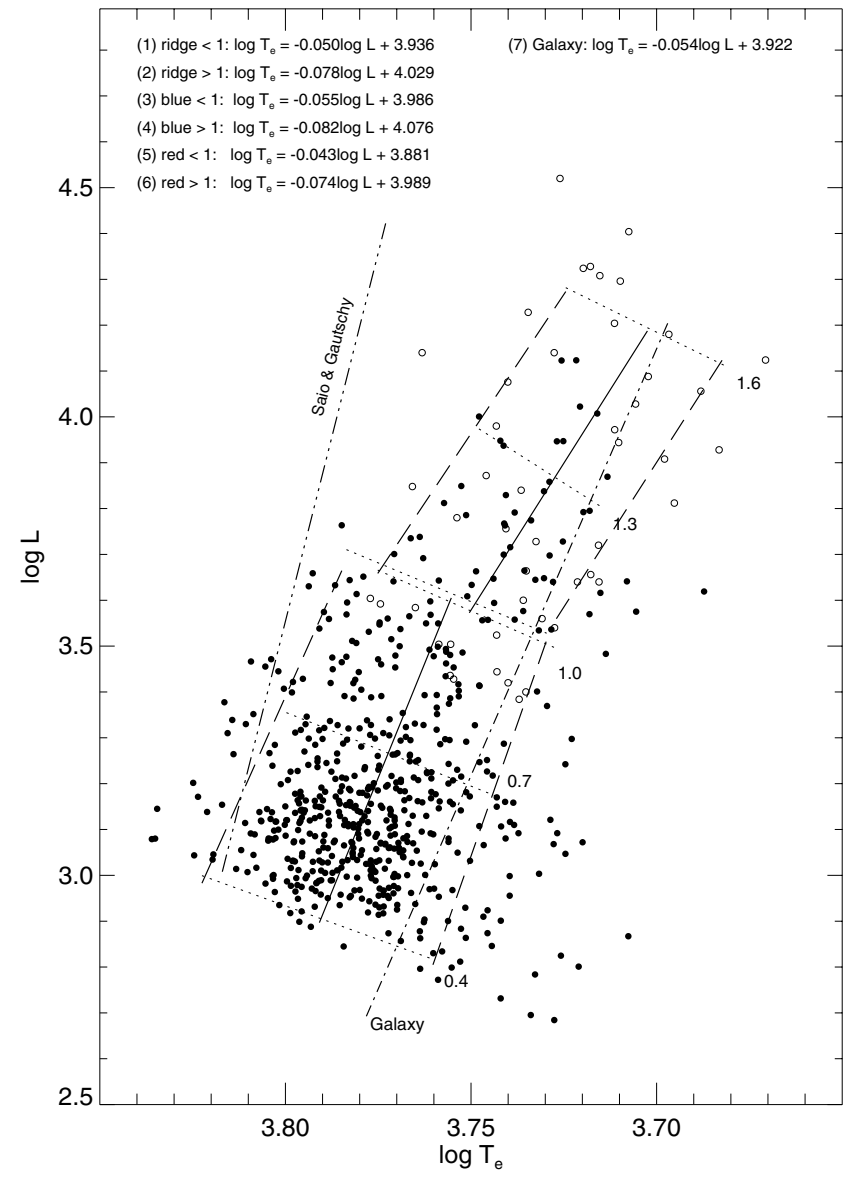

Fig. 20. The instability strip in the $\log L-\log T_{\mathrm{e}}$ plane. The individual Cepheids are shown. Symbols as in Fig. 1a. The ridge line (full line) and boundaries (dashed lines) are transformations of the corresponding lines in Fig. 8. The dashed-dotted line is the mean relation of the Galaxy. The theoretical line of Saio \& Gautschy (1998) is dasheddouble-dotted.

strip of $\Delta \log T_{\mathrm{e}}=0.08$, the reason for the temperature difference remains obscure. While it is true that the temperature of the blue edge of the strip depends on the metal abundance, $Z$, there is also a strong dependence on the helium abundance, $Y$.

The dependence of the blue edge temperature for fundamental mode Cepheids on $Y$ and $Z$ has been known since the earliest theoretical work on the low mass RR Lyrae variables by Iben \& Huchra (1971), Christy (1966), Tuggle \& Iben (1972), van Albada \& Baker (1971), and others into modern times. The earliest calculations of the $Z, Y$ dependence of the blue edge of the $L / T_{\mathrm{e}}$ strip for stars in the mass and luminosity range of the classical Cepheids was that of Iben \& Tuggle (1975). Their results remain nearly definitive today as shown by the following comparison.

SBT compared the position of the Iben and Tuggle blue edge $L\left(T_{\mathrm{e}}, Z, Y\right)$ locus with the modern Eq. (6) of Chiosi et al. (1992) for the $Z, Y$ blue edge dependencies and found identical temperatures to within about $\Delta \log T_{\mathrm{e}}=0.002$ when reduced to the same values of $Z$ and $Y$. Similar comparisons were made by SBT for the blue edge positions of Iben/Tuggle and Saio \& Gautschy (1998), and again were found to be nearly identical when reduced to same $Z$ and $Y$ compositions. 
These comparisons of Saio \& Gautschy with Iben \& Tuggle (1975) and Chiosi et al. (1992) were particularly important because Saio \& Gautschy used the new OPAL opacities by Rogers \& Iglesias (1992) as extended to lower temperatures by Alexander \& Ferguson (1994) whereas Iben \& Tuggle and Chiosi et al. used the earlier Los Almos Cox-Stewart opacities. Hence, differences in the adopted opacity tables have a negligible effect on the position of the blue edges of the instability strip.

Using Eq. (6) of Chiosi et al. for the blue edge as function of $Z$ and $Y$, and reading the mean mass/luminosity ratio at intermediate $Z$ and $Y$ values (in any case, the $T_{\mathrm{e}} / L$ blue edge position has only a small mass dependence), the temperature dependence on $Z$ and $Y$ is found to be

$\log T_{\mathrm{e}}=3.771+0.12 Y-0.66 Z$,

at $\log L=3.5\left(M_{\mathrm{bol}}=-4.0\right)$. Hence if $Y$ increases as $Z$ increases, due to the manufacture of helium with higher metallicity production, as all models of stellar nucleosynthesis require, the position of the blue edge would remain fixed (independent of $Y$ and $Z$ ) if $0.12 \Delta Y$ were to equal $0.66 \Delta Z$ (i.e. if $\Delta Y / \Delta Z=5.5$ ). There would then be no temperature shift in Fig. 20 due to different $Z$ metallicities.

However, the canonical change of $Y$ with $Z$ has the ratio of $\Delta Y / \Delta Z=2.2$ (VandenBerg in Sandage et al. 2003, hereafter SLV). The Padua astronomers, led by Chiosi, (see Fagotto et al. 1994 for the last of four papers on the evolutionary tracks for different compositions) use values close to 2.5 . The Geneva astronomers, led by Maeder, (the last paper of their four paper series is by Schaerer et al. 1993) used $\Delta Y / \Delta Z=3$. Hence, although there is compensation in the $T_{\mathrm{e}}$ value as $Y$ increases with $Z$, it is not complete if Eq. (50) is correct. If we use $\Delta Y / \Delta Z=2.2$, then for a change by a factor of 10 in $Z$ from 0.0188 (which is solar) to say 0.0019 , with $Y$ changing from 0.276 (solar) to 0.239 according to the prescription that $Y=0.235+2.2 Z$ given by SLV, then $\Delta \log T_{\mathrm{e}}=0.007$, with the low $Z$ case being hotter. This is much smaller than the requirement from Fig. 20 for $\Delta T_{\mathrm{e}}=0.03$ (although in the same direction), but even here the LMC metallicity is only smaller than the Sun by a factor of 4 not 10 . Hence, the compensation of $Y$ and $Z$ in Eq. (50) is the reason that the temperature of the blue edges for the variety of compositions used by the Padua astronomers and by Iben and Tuggle is virtually unchanged for variable $Z$. The hotter temperature for LMC Cepheids relative to those in the Galaxy is therefore not explained by Eq. (50).

Even if $Z$ changes by a factor of 10 in the above example with $Y$ kept constant for some reason, the change in $\log T_{\mathrm{e}}$ would only be $\Delta T_{\mathrm{e}}=0.01$, again smaller than a factor of two or three from what is required by Fig. 20.

Of course, a reverse sign variation of a decrease in $Y$ with increasing $Z$ would solve the dilemma, producing any hotter value for $T_{\mathrm{e}}$ for small $Z$ that the observations require (Fig. 20), but this is highly counter intuitive and entirely ad hoc. We are left with the dilemma that we do not have a reasonable explanation of the shift in $T_{\mathrm{e}}$ with lower metallicity in Fig. 20 based on any extant theoretical model. We cannot conclude that the variation is, in fact due, to metallicity, or at least not to metallicity alone.
Table 5. Predicted and observed slopes of the $\mathrm{P}-\mathrm{L}_{V}$ relation (see text).

\begin{tabular}{lccc}
\hline \hline Cepheids in & $c$ & $\begin{array}{c}\mathrm{d} M_{\mathrm{bol}} / \mathrm{d} \log P \\
\text { predicted }\end{array}$ & $\begin{array}{c}\mathrm{d} M_{V} / \mathrm{d} \log P \\
\text { observed }\end{array}$ \\
\hline Galaxy & -0.054 & -3.02 & $-3.09 \pm 0.09^{1}$ \\
LMC, $\log P<1$ & -0.050 & -3.09 & $-2.96 \pm 0.06^{2}$ \\
LMC, $\log P>1$ & -0.078 & -2.76 & $-2.57 \pm 0.10^{2}$ \\
\hline
\end{tabular}

${ }^{1}$ From Eq. (17); ${ }^{2}$ from Eqs. (12) and (13).

It remains to be shown that any change of the slope of the ridge line of the $\log T_{\mathrm{e}}-\log L$ relation translates into a change of slope of the P-L relation. In Paper I we have shown that the slope of the P-L relation is given by

$$
\begin{aligned}
\mathrm{d} M_{V} / \mathrm{d} \log P & \approx \mathrm{d} M_{\mathrm{bol}} / \mathrm{d} \log P \\
& =-2.5(0.84-0.68 a-3.48 c)^{-1},
\end{aligned}
$$

where $a$ is the coefficient of the $\log$ Mass- $\log L$ dependence, and $c$ is the slope of the $\log T_{\mathrm{e}}-\log L$ relation. This equation is based on a simple pulsation equation by van Albada \& Baker (1973) applicable to RR Lyrae stars and, as it turns out, is also an excellent equation for Cepheids in its coefficients from the $\log L, \log M$, and $\log T_{\mathrm{e}}$ dependences (see SBT for an extended discussion). We have used $a=0.30$, typical for Cepheids (cf. SBT, Tables 1-5). The coefficient $a$ enters only weakly into Eq. (51). The deduced values of $c$ are shown in Fig. 20 for the ridge lines of the short- and long-period LMC Cepheids and of Galactic Cepheids. The ridge line values are tabulated in Table 5 together with the predicted ridge line slopes from Eq. (51) and the corresponding observed values. The agreement between predicted and observed slopes is almost within the errors of the observed slopes. Allowing for some additional errors in the predicted slopes makes the agreement good. The most significant result is that the steep slope of the long-period LMC Cepheids in the $\log T_{\mathrm{e}}-\log L$ plane requires a flat slope of their P-L relation. This is observed, and is the main point of this paper. Hence, the different slope of the ridge line of the $\log L-\log T_{\mathrm{e}}$ relation between LMC and the Galaxy explains the different slopes to the P-L relation in Fig. 4 (lower right panel).

\section{Conclusions and summary}

The consequences of the differences in the slopes of the P-L relations for the Galaxy, LMC, and SMC weakens the hope of using Cepheids to obtain precision galaxy distances. Until we understand the reasons for the differences in the P-L relations and the shifts in the period-color relations, (after applying blanketing corrections for metallicity differences), we are presently at a loss to choose which of the several P-L relations to use (Galaxy, LMC, and SMC) for other galaxies.

Although we can still hope that the differences may yet be caused only by variations in metallicity, which can be measured, this can only be decided by future research such as survey programs to determine the properties of Cepheids in galaxies such as M33 and M 101 where metallicity gradients exist across the image. But until we can prove or disprove 
that metallicity difference is the key parameter, we must provisionally assume that this is the case, and use either the Galaxy P-L relations in Sect. 4.2.1 (Eqs. (16)-(18)), or the LMC P-L relations in Sect. 4.1 (Eqs. (7)-(15)), or those in the SMC from the forthcoming Paper III, in deriving galaxy distances from Cepheids. This has now in fact been done elsewhere for M 83 (Thim et al. 2003).

There are eleven principal research points in this paper. (1) The period-color (P-C) relations in $(B-V)^{0}$ and $(V-I)^{0}$ have significantly different slopes for periods smaller and larger than 10 days (Figs. 1a and 1b). The slope of the longer period Cepheids is steeper than those with $P<10$ days at the significance level of $3.4 \sigma$ in $(B-V)^{0}$ and $4.1 \sigma$ in $(V-I)^{0}$.

(2) The LMC Cepheids are bluer than Galactic Cepheids by $0 .{ }^{\mathrm{m}} 07$ in $(B-V)^{0}$ at $\log P=0.4$, increasing to 0.12 at $\log P=$ 1. The corresponding differences in $(V-I)^{0}$ are 0.03 and $0^{\mathrm{m}} .10$ (Eqs. (2)-(6) for LMC compared with Eqs. (3) and (5) of Paper I). Blanketing differences due to the lower metallicity of LMC accounts for only half of the observed color differences. A temperature difference (Figs. 3 and 20) accounts for the remainder, with the LMC Cepheids being hotter by between $350 \mathrm{~K}$ and $80 \mathrm{~K}$, depending on the period. Theoretical models of the position of the fundamental blue edge as functions of metallicity and helium abundance cannot account for the temperature difference (Eq. (50)) if $Z$ and $Y$ increase in lock step. An explanation is possible if $Y$ and $Z$ are anticorrelated (Sect. 8), but this is highly counterintuitive based on the models of nucleosynthesis.

(3) The P-L relations for LMC in $B, V$, and $I$ are all non-linear, each with a break at $\log P=1$ (Eqs. (10)-(15)). The slope differences are significant at the at least $3 \sigma$ level. The reality of the break in the P-L relations at 10 days is supported by the breaks in the P-C relations (item 1). Necessarily, breaks in the $\mathrm{P}-\mathrm{C}$ relations require breaks in the $\mathrm{P}-\mathrm{L}$ relations.

(4) The slopes of the LMC P-L relations in the $B, V$, and $I$ pass bands are different from the slopes of the corresponding relations in the Galaxy as updated in Sect. 4.2.1. For Cepheids with $\log P<1$ the P-L slope differences between LMC and the Galaxy vanish in $B$, but amount to $2 \sigma$ and $3 \sigma$ in $V$ and $I$. The significance of the slope differences is even larger for longperiod Cepheids with $\log P>1$, i.e. it increases from $3 \sigma$ in $B$ to $5 \sigma$ in $V$ and $I$. Since most available galaxy distances from Cepheids are based on long-period Cepheids ( $\log P_{\text {median }} \sim$ 1.4) the choice of which P-L relation is used has a nonnegligible effect on the derived absorption-corrected moduli by about 0.2 (cf. Eq. (40) in Paper I) or $\sim 10 \%$ in distance.

(5) The absolute magnitudes of LMC Cepheids are brighter than those in the Galaxy by 0.42 to 0.32 in $B, V$, and $I$ at $\log P=0.4$, becoming fainter by 0 . 06 in $V$ and by 0.14 in $I$ at $\log P=1.5$ (Eqs. (10)-(15) compared with (16)-(18)). All attempts to determine a Cepheid distance of LMC by means of a Galactic P-L relation (e.g. Fouqué et al. 2003; Groenewegen $\&$ Salaris 2003; Storm et al. 2004) are therefore frustrated. The resulting values of $(m-M)_{\mathrm{LMC}}^{0}$ would vary between $\sim 18.16$ and $\sim 18.63$ (still somewhat dependent on pass band) depending on whether Cepheids with $\log P=0.4$ or $\log P=1.5$ are compared. (The adopted LMC distance of 18.54 in the present paper rests on a compilation of distance determinations which are independent of the P-L relation of Cepheids; see Paper I, Table 6.) - There are now, however, good prospects to reestablish Cepheids as important distance indicators of LMC by means of their individual BBW distances, which depend little on metallicity. Work towards this aim is in progress (W. Gieren, private communication).

(6) The reality of the break in Cepheid properties at 10 days is shown in various correlations. (a) The Fourier components $R_{21}$ and $\Phi_{21}$, introduced by Simon \& Lee (1981), show discontinuities at 10 days (Fig. 6). (b) A similar discontinuity exists in the period-amplitude relation, both for LMC and the Galaxy (Fig. 7). (c) The slope of the ridge-line of the color-magnitude diagram for the instability strip (Fig. 8) changes at 10 days, as in the theoretical HR $\left(L / T_{\mathrm{e}}\right)$ diagram (Fig. 20). (d) Changes at 10 days also occur in other correlations such as the slopes of the lines of constant period (Fig. 10 and Sect. 6.2), the character of the color-amplitude correlation in the period range of 10 to 20 days (Figs. 11 and 12), and in the $R_{21}$ vs. $\log P$ relation with the data binned by absolute magnitude (Fig. 15).

(7) The loci of constant period in the CMD (Fig. 8) slope toward fainter magnitudes as the color changes across the strip from blue to red. Hence, at a given period there is a variation in absolute magnitude causing the systematic scatter in the P-L relations in $B, V$, and $I$. The mapping of this effect is particularly strong here using the LMC data because the data base over the entire period range from 2 to 40 days is superbly large from the OGLE project. The slopes of the lines of constant period in the $M_{V}$ P-L relation average 1.8 in $(B-V)^{0}$ (but vary with period) and 2.4 in $(V-I)^{0}$ (Fig. 9 and Eqs. (27)-(29)). The slope of the constant-period lines is of course steeper than this in the $M_{B}-(B-V)$ plane and more shallow in the $M_{I}-(V-I)$ plane (Eqs. (43) and (44)), showing why the scatter in the P-L relations becomes progressive smaller from $B$, through $V$, to $I$, as was originally shown (Sandage 1958, 1972) as due to the finite color width of the instability strip.

(8) The large data base permits a high-weight mapping of several Cepheid characteristics with position in the strip. The data binned in narrow period intervals from 3 to 40 days, (i.e. along lines of constant period in the CMD), show (Fig. 11) that, except near 10 days, the amplitude is largest at the blue edge of the strip, just as for the RR Lyrae stars in the low mass instability strip peculiar to them. The analogy with the RR Lyrae stars is stronger when the data are binned in narrow intervals of absolute magnitude (Fig. 12) similar to the case of RR Lyrae variables in globular clusters. Figure 12 shows that the correlation of light-curve amplitude with color (at the relevant absolute magnitude from -2.75 to brighter than -4.75 ) is in the sense that largest amplitude occurs at the bluest color for Cepheids fainter than $M_{V}=-4.25$ (periods smaller than 7 days), the trend reverses for periods of 10 to 15 days $\left(-4.25<M_{V}<\right.$ -4.75) and reverts to the original sense for $P>20$ days as was earlier found by Sandage \& Tammann (1971) from a much smaller data sample.

(9) The Fourier coefficient $R_{12}$ varies systematically with amplitude and in color across the strip as shown in Fig. 13, binned by period and in Fig. 14, in analogy with the RR Lyrae correlations, binned by absolute magnitude. Large $R_{21}$ means that the second term of the Fourier series, $\sin 2 x$, is large, showing 
that the light curve is highly peaked (approaching a saw tooth for $R_{21}=0.5$; Sect. 6.4). Small $R_{21}$ stands for nearly symmetrical light curves, which, by Fig. 13, occur at small amplitude in all period ranges.

Hence, Figs. 13 and 14 show that the light curve shapes are highly peaked toward the blue edge of the instability strip, becoming nearly sinusoidal (and of small amplitude) toward the red. $R_{12}$ varies systematically across the strip. This is an important result concerning the physics of pulsation. Large amplitude with its near saw-tooth shape (small interval in phase between minimum and maximum light) means that the pulsation is more strongly driven into the non-linear regime at the blue edge than toward the red edge. Hence, the prediction is that the PV work diagram in the pulsation thermodynamics must have a larger non-dissipative area for Cepheids near the blue edge than near the red edge (cf. Christy 1966).

(10) By necessity, from these correlations of $R_{21}$ with color (Figs. 13 and 14), and from the correlations of color and period, (Fig. 16) there must be a correlation of $R_{21}$ with period at constant absolute magnitude. This is confirmed in Fig. 15. The reason is obvious (by inspecting the CMD of Fig. 8), once it is known that $R_{21}$ varies systematically with color across the strip.

(11) Application of the PLC relation to the entirety of the OGLE LMC data gives the relative distance to each individual LMC Cepheid. This permits determination of the orientation of the bar in the line of sight. The eastern edge of the bar is only 0.03 closer than the western edge (Fig. 19), confirming earlier conclusions by Welch et al. (1987) and Caldwell \& Laney (1991).

Finally we reiterate that the unexpected slope difference between the P-L relations particularly of long-period Cepheids in the Galaxy and LMC can be understood as the consequence of the different gradients in the luminosity-temperature diagram, yet the reason why these gradients are different remains unknown.

Acknowledgements. G.A.T. and B.R. thank the Swiss National Science Foundation for valuable support. The authors thank Dres. D. Bersier, R. Buser, A. Gautschy, S. Kanbur, C. Ngeow, M. Samland, N. Simon, A. Udalski, and N. Walborn for helpful discussions and informations. Much of this paper was written while G.A.T. was a visitor at the Space Telescope Science Institute in Baltimore. He thanks the Director and the Staff for their hospitality and for a most stimulating period of time.

\section{References}

Alexander, D. R., \& Ferguson, J. W. 1994, ApJ, 437, 879

Alibert, Y., Baraffe, I., Hauschildt, P., \& Allard, F. 1999, A\&A, 344, 551

Allen, P. D., \& Shanks, T. 2004, MNRAS, 347, 1011

Antonello, E., Fugazza, D., \& Mantegazza, L. 2002, A\&A, 388, 477

Baraffe, I., \& Alibert, Y. 2001, A\&A, 371, 592

Barnes, T., Jeffreys, W., Berger, J., et al. 2003, ApJ, 592, 539

Becker, S. A., Iben, I., \& Tuggle, R. S. 1977, ApJ, 218, 633

Benedict, G. F., McArthur, B. E., Fredrick, L. W., et al. 2002, AJ, 124, 1695

Berdnikov, L. N., Dambis, A. K., \& Voziakova, O. V. 2000, A\&AS, 143,211
Berdnikov, L. N., \& Ivanov, G. R. 1986, Ap\&SS, 125, 201

Buchler, J. R., Goupil, M. J., \& Piciullo, R. 1997, ApJ, 491, L99

Caldwell, J. A. R., \& Coulson, I. M. 1985, MNRAS, 212, 879

Caldwell, J. A. R., \& Coulson, I. M. 1986, MNRAS, 218, 223

Caldwell, J. A. R., Coulson, I. M., Jones, J. H. S., Black, C. A., \& Feast, M. W. 1986, MNRAS, 220, 671

Caldwell, J. A. R., \& Laney, C. D. 1991, in The Magelanic Clouds, ed. R. Haynes, \& D. Milne, IAU Symp., 148 (Dordrecht: Kluwer), 249

Caputo, F., Marconi, M., \& Musella, I. 2000, A\&A, 354, 610

Chiosi, C., Wood, P., Bertelli, G., \& Bressan, A. 1992, ApJ, 387, 320

Christy, R. F. 1966, ApJ, 144, 108

Fagotto, F., Bressan, A., Bertelli, G., \& Chiosi, C. 1994, A\&AS, 105, 39

Feast, M. W. 1999, PASP, 111, 775

Feast, M. W. 2002, MNRAS, 337, 1035

Feast, M. W., \& Catchpole, R. M. 1997, MNRAS, 286, 1

Fernie, J. D., \& Ehlers, P. 1999, AJ, 117, 1563

Fouqué, P., Storm, J., \& Gieren, W. 2003, Lect. Notes Phys., 635, 21

Gieren, W. P., Fouqué, P., \& Gómez, M. 1998, ApJ, 496, 17

Groenewegen, M. A. T., \& Oudmaijer, R. D. 2000, A\&A, 356, 849

Groenewegen, M. A. T., \& Salaris, M. 2003, A\&A, 410, 887

Hertzsprung, E. 1924, BAN, 2, 83

Hertzsprung, E. 1926, BAN, 3, 115

Hodge, P. W. 1972, BAAS, 4, 223

Hofmeister, E. 1967, Z. Astrophys., 65, 194

Hoyle, F., Shanks, T., \& Tanvir, N. R. 2003, MNRAS, 345, 269

Iben, I., \& Huchra, J. 1971, A\&A, 14, 293

Iben, I., \& Tuggle, R. S. 1975, ApJ, 197, 39

Kanbur, S. M., \& Ngeow, C.-C. 2004, MNRAS, 350, 962

Kervella, P., Nardetto, N., Bersier, D., Mourard, D., \& Coudé du Foresto, V. 2004, A\&A, 416, 941

Kovács, G. 2003, MNRAS, 342, L58

Kraft, R. P. 1961, ApJ, 134, 616

Lane, B., Creech-Eakman, M., \& Nordgren, T. 2002, ApJ, 573, 330

Laney, C. D., \& Stobie, R. S. 1986, MNRAS, 222, 449

Laney, C. D., \& Stobie, R. S. 1994, MNRAS, 266, 441

Lanoix, P., Paturel, G., \& Garnier, R. 1999, MNRAS, 308, 969

Ludendorff, von H. 1919, AN, Nr. 5006, Vol. 209, 218

Madore, B. F. 1985, in Cepheids: Theory and Observations, ed. B. F.

Madore, IAU Coll., 82 (Cambridge: Univ. Cambridge Press), 166

Madore, B. F., \& Freedman, W. L. 1991, PASP, 103, 933

Makarov, V. V. 2002, AJ, 124, 3299

Martin, W. L. 1980, South Afr. Astr. Obs. Circ. 1, No. 5, 172

Martin, W. L., Warren, P. R., \& Feast, M. W. 1979, MNRAS, 188, 139

Moskalik, P., Buchler, J. R., \& Marom, A. 1992, ApJ, 385, 685

Narayanan, V. K., \& Gould, A. 1999, ApJ, 523, 328

Ngeow, C.-C., Kanbur, S. M., Nikolaev, S., Tanvir, N. R., \& Hendry, M. A. 2003, ApJ, 586, 959

Ngeow, C.-C., \& Kanbur, S. M. 2004, MNRAS, 349, 1130

Nordgren, T. E., Lane, B. F., Hindsley, R. B., \& Kervella, P. 2002, AJ, 123,3380

O’Dell, M. A., Hendry, M. A., \& Collier Cameron, A. 1994, MNRAS, 268,181

Pan, X., Shao, M., \& Kulkarni, S. R. 2004, Nature, 427, 326

Parodi, B. R., Saha, A., Sandage, A., \& Tammann, G. A. 2000, ApJ, 540,634

Paturel, G., Teerikorpi, P., Fouqué, P., et al. 2002, A\&A, 389, 19

Payne-Gaposchkin, C. H. 1974, Smithsonian Contr. Ap., No. 16

Pinsonneault, M. H., Stauffer, J., Soderblom, D. R., King, J. R., \& Hanson, R. B. 1998, ApJ, 504, 170

Rogers, F. J., \& Iglesias, C. A. 1992, ApJS, 79, 507

Saha, A., Sandage, A., Tammann, G. A., et al. 2001, ApJ, 562, 314 
Saio, H., \& Gautschy, A. 1998, ApJ, 498, 360

Sandage, A. 1958, ApJ, 127, 513

Sandage, A. 1972, QJRAS, 13, 202

Sandage, A. 1988, PASP, 100, 935

Sandage, A., Bell, R. A., \& Tripicco, M. J. 1999, ApJ, 522, 250 (SBT)

Sandage, A., \& Gratton, L. 1963, in Stellar Evolution, Proc. of the XXVIIIth Course of the International School of Physics "Enrico Fermi", ed. L. Gratton (New York: Academic Press), 11

Sandage, A., Lubin, L. M., \& VandenBerg, D. A. 2003, PASP, 115, 1187 (SLV)

Sandage, A., \& Tammann, G. A. 1968, ApJ, 151, 531

Sandage, A., \& Tammann, G. A. 1969, ApJ, 157, 683

Sandage, A., \& Tammann, G. A. 1971, ApJ, 167, 293

Schaerer, D., Charbonnel, C., Meynet, G., Maeder, A., \& Schaller, G. 1993, A\&AS, 102, 339

Schaltenbrand, R., \& Tammann, G. A. 1970, A\&A, 7, 289

Schaltenbrand, R., \& Tammann, G. A. 1971, A\&AS, 4, 265

Sebo, K. M., Rawson, D., Mould, J., et al. 2002, ApJS, 142, 71

Simon, N. R., \& Lee, A. S. 1981, ApJ, 248, 291

Simon, N. R., \& Moffett, T. J. 1985, PASP, 97, 1078

Simon, N. R., \& Young, T. S. 1997, MNRAS, 288, 267
Stello, D., \& Nissen, P. E. 2001, A\&A, 374, 105

Storm, J., Carney, B. W., Gieren, W. P., et al. 2004, A\&A, 415, 531

Tammann, G. A., \& Reindl, B. 2002, Ap\&SS, 280, 165

Tammann, G. A., Reindl, B., Thim, F., Saha, A., \& Sandage, A. 2002, in A New Era in Cosmology, ed. T. Shanks, \& N. Metcalfe, ASP Conf. Ser., 283, 258

Tammann, G. A., Sandage, A., \& Reindl, B. 2003, A\&A, 404, 423 (Paper I)

Teerikorpi, P., \& Paturel, G. 2002, A\&A, 381, 37

Thim, F., Tammann, G. A., Saha, A., et al. 2003, ApJ, 590, 256

Tuggle, R. S., \& Iben, I. 1972, ApJ, 178, 455

Turner, D. G., \& Burke, J. F. 2002, AJ, 124, 2931

Udalski, A., Szymanski, M., Kubiak, M., et al. 1999a, AcA, 49, 201

Udalski, A., Soszynski, I., Szymanski, M., et al. 1999b, AcA, 49, 223

Udalski, A., Soszynski, I., Szymanski, M., et al. 1999c, AcA, 49, 437

van Albada, T. S., \& Baker, N. 1971, ApJ, 169, 311

van Albada, T. S., \& Baker, N. 1973, ApJ, 185, 477

Welch, D. L., McLaren, R. A., Madore, B. F., \& McAlary, C. W. 1987, ApJ, 321, 162

Woosley, S. E., \& Weaver, T. A. 1986, ARA\&A, 24, 205

Zakrzewski, B., Ogloza, W., \& Moskalik, P. 2000, AcA, 50, 387 